\title{
Long noncoding RNA SGO1-AS1 inactivates TGF $\beta$ signaling by facilitating TGFB1/2 mRNA decay and inhibits gastric carcinoma metastasis
}

\author{
Donglan Huang ${ }^{1+}$ (D), Ke Zhang ${ }^{1 \dagger}$, Wenying Zheng ${ }^{1}$, Ruixin Zhang ${ }^{1}$, Jiale Chen ${ }^{1}$, Nan Du ${ }^{2}$, Yuanyuan Xia ${ }^{3}$, \\ Yan Long ${ }^{4}$, Yixue $\mathrm{Gu}^{1^{*}}$, Jianhua $\mathrm{Xu}^{5^{*}}$ and Min Deng ${ }^{1 *}$
}

\begin{abstract}
Background: Although thousands of long noncoding RNAs (IncRNAs) have been annotated, only a few IncRNAs have been characterized functionally. In this study, we aimed to identify novel IncRNAs involved in the progression of gastric carcinoma (GC) and explore their regulatory mechanisms and clinical significance in GC.

Methods: A IncRNA expression microarray was used to identify differential IncRNA expression profiles between paired GCs and adjacent normal mucosal tissues. Using the above method, the IncRNA SGO1-AS1 was selected for further study. Quantitative reverse transcription polymerase chain reaction (qRT-PCR) and in situ hybridization (ISH) were performed to detect SGO1-AS1 expression in GC tissues. Gain-of-function and loss-of-function analyses were performed to investigate the functions of SGO1-AS1 and its upstream and downstream regulatory mechanisms in vitro and in vivo.

Results: SGO1-AS1 was downregulated in gastric carcinoma tissues compared to adjacent normal tissues, and its downregulation was positively correlated with advanced clinical stage, metastasis status and poor patient prognosis. The functional experiments revealed that SGO1-AS1 inhibited GC cell invasion and metastasis in vitro and in vivo. Mechanistically, SGO1-AS1 facilitated TGFB1/2 mRNA decay by competitively binding the PTBP1 protein, resulting in reduced TGF $\beta$ production and, thus, preventing the epithelial-to-mesenchymal transition (EMT) and metastasis. In addition, in turn, TGF $\beta$ inhibited SGO1-AS1 transcription by inducing ZEB1. Thus, SGO1-AS1 and TGF $\beta$ form a doublenegative feedback loop via ZEB1 to regulate the EMT and metastasis.
\end{abstract}

Conclusions: SGO1-AS1 functions as an endogenous inhibitor of the TGF $\beta$ pathway and suppresses gastric carcinoma metastasis, indicating a novel potential target for GC treatment.

Keywords: Gastric carcinoma, Metastasis, IncRNA, SGO1-AS1, TGF $\beta$, ZEB1

*Correspondence: guyx189@163.com; jhxu1976@gzucm.edu.cn; mindeng@gzhmu.edu.cn

${ }^{\dagger}$ Donglan Huang and Ke Zhang contributed equally to this work. 1 Affiliated Cancer Hospital \& Institute of Guangzhou Medical University,

No.78 Hengzhigang Road, Guangzhou 510095, Guangdong, China ${ }^{5}$ Laboratory of Oncology Science and Molecular Biology, ShunDe Hospital of Guangzhou University of Chinese Medicine, No.12 Jinsha Avenue, Shunde District, Foshan 528333, Guangdong, China

Full list of author information is available at the end of the article

\section{Background}

Gastric carcinoma is one of the most common malignancies worldwide and the fourth leading cause of cancer death [1]. Approximately $40 \%$ of patients with gastric carcinoma present with metastases, and only approximately $5 \%$ of these patients exhibit 5-year survival [2]. The prognosis of GC patients with metastatic disease remains poor due to the lack of effective therapies. New therapeutic options will become available only if we improve our original author(s) and the source, provide a link to the Creative Commons licence, and indicate if changes were made. The images or other third party material in this article are included in the article's Creative Commons licence, unless indicated otherwise in a credit line to the material. If material is not included in the article's Creative Commons licence and your intended use is not permitted by statutory regulation or exceeds the permitted use, you will need to obtain permission directly from the copyright holder. To view a copy of this licence, visit http://creativecommons.org/licenses/by/4.0/. The Creative Commons Public Domain Dedication waiver (http://creativeco mmons.org/publicdomain/zero/1.0/) applies to the data made available in this article, unless otherwise stated in a credit line to the data. 
understanding of the mechanisms underlying metastatic spread.

LncRNAs are transcripts longer than 200 nucleotides without protein-coding potential [3]. Tens of thousands of lncRNAs are expressed in human cells, but the function of most lncRNAs remains unknown [4]. An increasing number of studies have demonstrated the importance of lncRNAs for regulating a wide range of processes, including development, differentiation, cell proliferation, cell death and cancer development $[5,6]$. Recently, several GC-implicated lncRNAs have been identified, and their functions and mechanisms have been clarified [711]. For instance, the lncRNA GClnc1 promotes gastric carcinogenesis and may act as a scaffold for WDR5 and KAT2A complexes to specify the histone modification pattern [9]. The IncRNA GMAN enhances the translation of ephrin A1 mRNA by competitively binding GMANAS and, thus, promotes GC invasion and metastasis [10]. However, the well-characterized IncRNAs involved in GC are merely the tip of the iceberg, and an even larger number remain unknown.

Here, we demonstrate that the lncRNA SGO1-AS1 (also known as SGOL1-AS1), which is downregulated in gastric carcinoma and associated with tumor progression and patient prognosis, prevents gastric carcinoma EMT, invasion and metastasis in vitro and in vivo. Mechanistically, SGO1-AS1 reduces the stability of TGFB1/2 mRNA by competitively binding the PTBP1 protein, resulting in reduced TGF $\beta$ production. In turn, TGF $\beta$ inhibits SGO1AS1 transcription by inducing ZEB1. Thus, in this study, we identified a novel metastasis-suppressive lncRNA, i.e., SGO1-AS1, with crucial biological, mechanistic and clinical impacts on GC that mediates a double-negative feedback loop with TGF $\beta$ via ZEB1.

\section{Methods}

\section{Clinical specimens}

Five pairs of snap-frozen GC tissues and matched adjacent normal mucosa tissues were obtained for the lncRNA microarray analysis. Furthermore, the following two cohorts of frozen samples were collected for the qRT-PCR assay: a small GC cohort (Cohort 1) containing 18 pairs of GC tissues and corresponding adjacent normal mucosa tissues to confirm 13 lncRNAs with more than a 4-fold difference in the microarray analysis and a large GC cohort (Cohort 2) including 92 pairs of GC tissues and matched adjacent normal samples to detect the expression levels of SGO1-AS1, TGFB1/2 and ZEB1. Additionally, GC tissue microarrays containing $95 \mathrm{GC}$ tissues and 80 adjacent tissues (Cohort 3) were included in this study for the ISH analysis. All tissues were collected immediately after surgery from the Affiliated Cancer Hospital of Guangzhou Medical University
(Guangzhou, Guangdong, China). All procedures carried out in this research involving human participants were performed in accordance with the ethical standards of the Institutional Review Board of the Affiliated Cancer Hospital of Guangzhou Medical University. The clinical and histopathological characteristics of the patients are described in Additional file 1: Table S1-2.

\section{Microarray analysis}

The total RNA was extracted from 5 paired GC tissues and corresponding adjacent normal mucosa tissues using TRIzol reagent (Invitrogen, Carlsbad, CA, USA) according to the manufacturer's instructions. The total RNA was amplified and reverse-transcribed into fluorescent cDNA. Then, the labeled cDNA was hybridized onto the LncRNA+mRNA Human Gene Expression Microarray V4.0 (Agilent, Palo Alto, CA), and after washing, the arrays were scanned with an Agilent Scanner G2565CA (Agilent). Agilent Feature Extraction software (version 10.7.3.1) was used to analyze the acquired array images and the Agilent qRT-PCR results. The data are available via Gene Expression Omnibus (GEO) under accession number GSE157289.

\section{qRT-PCR}

The total RNA was isolated from patient tissues and cultured cells using TRIzol reagent (Invitrogen), and cDNA was synthesized using a PrimeScript RT Reagent Kit (Takara, Otsu, Japan). Subsequently, quantitative polymerase chain reaction (qPCR) analyses were performed using a SYBR Premix Ex Taq Kit (Applied Biosystems, Foster City, CA, USA). $\beta$-actin was used as the endogenous control to normalize gene expression. The mRNA expression of SGO1-AS1, TGFB1, TGFB2 and PTBP1 in the human tissues is presented as $-\Delta \mathrm{Ct}$, and the gene expression in cells with different treatments is presented as $2^{-\Delta \Delta C t}$. The $\Delta C t$ was calculated by subtracting the $\mathrm{Ct}$ of $\beta$-actin from the Ct of the gene of interest. The $\Delta \Delta \mathrm{Ct}$ was calculated by subtracting the $\Delta \mathrm{Ct}$ of the control sample from the $\Delta \mathrm{Ct}$ of the treatment sample. The primer sequences for each gene are provided in Additional file 1: Table S3.

\section{In situ hybridization (ISH)}

The ISH analysis was performed using a kit from Boster (Wuhan, Hubei, China). Tissue microarray slides were deparaffinized, digested with proteinase $\mathrm{K}$, hybridized with DIG-labeled probes for SGO1-AS1 and U6 (positive control) at $52^{\circ} \mathrm{C}$ overnight and subsequently visualized with an anti-DIG-POD antibody and DAB complex. The SGO1-AS1 probe was 5'-CCGCCTCCCAGCCAA CCAATGGAGGAGCGAGGCG-3'. The results were evaluated by two individuals in a blinded fashion, and the 
SGO-AS1 expression levels were quantified according to its positive percentage and staining intensity.

\section{Rapid amplification of CDNA ends (RACE) analysis}

We used 5'-RACE and 3'-RACE analyses to determine the transcriptional initiation and termination sites of SGO1-AS1 using a SMARTer ${ }^{\mathrm{TM}}$ RACE cDNA Amplification Kit (Clontech, Palo Alto, CA, USA) following the manufacturer's instructions. Nested PCR products were cloned into the pMD20-T vector and then sequenced. The sequences of the SGO1-AS1-specific primers used in the nested PCR of the RACE assay are shown in Additional file 1: Table S4.

\section{Subcellular fractionation}

Nuclear and cytoplasmic separation was performed using a PARIS Kit (Life Technologies, USA) according to the manufacturer's instructions, and then, a qRT-PCR analysis was conducted.

\section{Cell culture}

The GC cell lines SGC7901, BGC823, AGS, MGC803, MKN45 and MKN28 were obtained from the Chinese Academy of Medical Science (Beijing, China), and the gastric epithelial cell line GES-1 was obtained from the Beijing Institute for Cancer Research (Beijing, China). The GC cell line NCI-N87 and the HEK293T cell line were obtained from the American Type Culture Collection (Manassas VA, USA). The cell lines involved in our experiments were reauthenticated by a short tandem repeat analysis every 6 months after resuscitation in our laboratory. These cells were cultured in Dulbecco's modified Eagle's medium (DMEM, Gibco, USA) supplemented with $10 \%$ fetal bovine serum (Gibco) at $37^{\circ} \mathrm{C}$ in $5 \% \mathrm{CO}_{2}$.

\section{RNAi, plasmid construction and cell transfection}

The recombinant lentiviral vectors used for SGO1-AS1 overexpression or knockdown were purchased from RiboBio (Guangzhou, Guangdong, China), and the PTBP1 short hairpin RNA (shRNA) lentiviral vectors were obtained from GeneChem (Shanghai, China). The target sequences for SGO1-AS1 and PTBP1 were as follows: shAS1\#1, 5'-GCTATCTTCCTCCTCCTCACA$3^{\prime}$; shAS1\#2, 5'-CTACCGCCGCCACATTCGAAA-3'; shAS1\#3, 5'-GCCTCCCTCTTGTGAGAAGAA-3'; shAS1\#4, 5'-AGCTTGCAACGCGGAAGCAGC-3'; and shPTBP1， 5'-GCGGCCAGCC CATCTACATC-3'. To establish the cell lines that stably overexpress or deplete SGO1-AS1, SGC-7901 cells were infected with recombinant SGO1-AS1 lentiviruses, while MKN28 cells were infected with SGO1-AS1 shRNA lentiviruses. Then, the infected cells were selected with $1 \mathrm{mg} / \mathrm{L}$ puromycin (InvivoGen, San Diego, CA, USA) for 2 weeks to obtain cells with stable overexpression or knockdown of SGO1-AS1. siRNAs targeting ZEB1 or AGO2 were designed and synthesized by Sangon Biotech (Shanghai, China), and their sequences are as follows: siZEB1\#1 sense, GGC AAGUGUUGGAGAAUAAUC, antisense, UUAUUC UCCAACACUUGCCUU; siZEB1\#2 sense, GGACAG CACAGUAAAUCUACA, antisense, UAGAUUUAC UGUGCUGUCCUG; siAGO2 sense, GGUUGAUAC UUAAGCUCUAUU, antisense, UAGAGCUUAAGU AUCAACCUG. To construct the reporter vectors for SGO1-AS1 promoter activity, the wild-type SGO1-AS1 promoter sequence ( $1 \mathrm{~kb}$ sequence upstream of the transcription start site) and its ZEB1-binding site mutated sequences were chemosynthesized by Huada (Shenzhen, Guangdong, China) and inserted into the vector pGL3 basic (Promega) upstream of the firefly luciferase gene.

\section{PTBP1 knockout by CRISPR/Cas9}

A small guide RNA (sgRNA) targeting the genome sequence of PTBP1 was cloned into LentiCRISPRv2 (Addgene), and lentivirus particles were generated by cotransfecting the recombinant vector and packaging plasmids into HEK293T packaging cells. MKN28 cells were infected with lentiviruses, and single cells were isolated $48 \mathrm{~h}$ after infection by FACS (BD FACS Aria III) into 96-well plates. Independent clones were allowed to grow for 3 weeks. The PTBP1 knockout cells were identified by Western blotting and targeted Sanger sequencing. The sgRNA targeting PTBP1 was 5'-CAGAGCAGACCC GCGGGGGA-3'.

\section{Western blotting analysis}

The Western blotting analysis was performed using standard procedures. The following primary antibodies were used in the experiments: anti-PTBP1 antibody (Cell Signaling Technology, Beverly, MA, USA), anti-PTBP2 antibody (Abcam, Cambridge, UK), anti-PTBP3 antibody (Sigma-Aldrich, St. Louis, MO, USA), anti-HNRNPK antibody (Abcam), anti-HNRNPM antibody (SigmaAldrich), anti-FUBP3 antibody (Abcam), anti-CPSF2 antibody (Abcam), anti-G3BP2 antibody (Atlas Antibodies), anti-TGF $\beta 1$ antibody (Proteintech Group), antiTGF $\beta 2$ antibody (Abcam), anti-p-SMAD2 antibody (Cell Signaling Technology), anti-SMAD2 antibody (Cell Signaling Technology), anti-p-SMAD3 antibody (Cell Signaling Technology), anti-SMAD3 antibody (Cell Signaling Technology), anti-SMAD5 antibody (Abcam), anti-ID2 antibody (Abcam), anti-ZEB1 antibody (Abcam), antiSNAI antibody (Abcam), anti-E-cadherin antibody (Proteintech Group), anti-Vimentin antibody (Cell Signaling Technology), anti-N-cadherin antibody (Cell Signaling Technology) and anti-GAPDH antibody (Sigma-Aldrich). The blots were incubated with a goat anti-rabbit or 
anti-mouse secondary antibody (Sigma-Aldrich) and visualized with a commercial ECL kit (Pierce, Rockford, IL).

\section{RNA pull-down assay}

The RNA pull-down assays were carried out as previously described. Briefly, the SGO1-AS1 sequences were cloned into the pMD20-T vector with the T7 promoter and transcribed in vitro with biotin RNA labeling mix and T7 RNA polymerase (Invitrogen) according to the manufacturer's instructions. The RNA pulldown assay was performed using a Pierce Magnetic RNA-Protein Pull-Down Kit (Millipore, Bedford, MA, USA) according to the manufacturer's instructions. Finally, the retrieved proteins were measured using sodium dodecyl sulfatepolyacrylamide gel electrophoresis (SDS PAGE) gels for mass spectrometry or a Western blot analysis.

\section{RNA immunoprecipitation (RIP) assay}

The RIP assays were performed using a Magna RIP RNABinding Protein Immunoprecipitation Kit (Millipore, Bedford, MA, USA) according to the manufacturer's instructions. Briefly, $100 \mu \mathrm{L}$ of cell extract were incubated with magnetic bead-antibody complex. Antibodies were used for RIP, and IgG served as a negative control. The precipitated RNAs were isolated using TRIzol (Invitrogen) for the RNA sequencing (RNA-seq) and qRT-PCR analyses.

\section{Chromatin immunoprecipitation (ChIP) assay}

The ChIP assays were performed using a Chromatin Immunoprecipitation Assay Kit (Millipore, Bedford, MA, USA). MKN28 cells were exposed to TGF $\beta 1$ or vehicle for $24 \mathrm{~h}$ and then crosslinked, lysed and sonicated. Immunoprecipitation was performed using an anti-ZEB1 antibody (Abcam, Cambridge, UK) and IgG. The precipitated DNA was quantified using qPCR and normalized to the respective $2 \%$ input.

\section{RNA-seq analysis}

To identify the differentially expressed genes upon PTBP1 knockout, the total RNA was isolated from the PTBP1 knockout or control MKN28 cells using TRIzol reagent, and PolyA RNA was subsequently purified from the total RNA using the NEBNext Poly(A) mRNA Magnetic Isolation Module. RNA-seq was performed to detect the mRNA expression profiles at GENTED (Shanghai, China) using HiSeq3000 (Illumina, USA). The differentially expressed genes with a fold change $>2$ and a $P$-value $<0.05$ were selected. To reveal the PTBP1bound mRNAs, RIP experiments were conducted using a PTBP1 antibody (Cell Signaling Technology) or IgG. The total RNA was isolated with TRIzol (Invitrogen), and ribosomal RNA was removed from the total RNA.
RNA-seq was performed at CLOUDSEQ (Shanghai, China) using HiSeq3000 (Illumina, USA). The data are available via GEO under accession numbers GSE157582 and GSE157941.

\section{Luciferase reporter assay}

HEK293T cells were seeded in 24-well plates and transfected with SGO1-AS1 promoter reporter constructs with wild-type or mutated ZEB1 binding sites. The pTKCluc vector was used as an internal transfection control. The transfected cells were treated with TGF $\beta 1$ ( $5 \mathrm{ng} /$ $\mathrm{mL}$ ) or vehicle control for $48 \mathrm{~h}$, and firefly and Renilla luciferase activities were measured using a Dual-Luciferase Reporter Assay System (Promega) following the manufacturer's instructions. The SBE4 promoter luciferase reporter vector (Addgene) was transfected into the PTBP1 knockout or control MKN28 cells. In addition, HEK293T cells were transfected with SBE4 promoter reporter vectors and then treated with conditioned medium from cells with SGO1-AS1 knockdown or overexpression. The firefly and Renilla luciferase activities were measured $48 \mathrm{~h}$ after the transfection using a dual luciferase system.

\section{Cell invasion, migration and proliferation assays}

For the cell invasion assay, starved cells suspended in serum-free DMEM were seeded into the upper chamber with Matrigel in the insert of a 24-well culture plate (Corning Costar). Medium containing 15\% fetal bovine serum was added to the lower compartment as a chemoattractant. After incubation for $48 \mathrm{~h}$, the invasive cells adhering to the lower membrane of the inserts were fixed, stained, counted and imaged. The cell migration ability was measured using a wound-healing assay. The cells were placed in 6-well plates and cultured until reaching $90 \%$ confluence. An artificial scratch was created using a $10 \mu \mathrm{L}$ pipette tip, and the cells were cultured in serum-free medium for $36 \mathrm{~h}$ or $48 \mathrm{~h}$. Wound closure images were captured in the same field under magnification. Cell proliferation was examined using cell counting. The cells were seeded into 6 -well plates, and the cell numbers were counted after 1, 2, 3, 4, 5, 6 and 7 days of culture in DMEM supplemented with 10\% fetal bovine serum using a Coulter Counter.

\section{Sphere culture}

Cells were seeded into ultralow attachment 6-well plates (Corning Costar) and cultured in DMEM/F12 medium (Gibco) supplemented with 2\% B27 (Life Technologies), $20 \mathrm{ng} / \mathrm{ml}$ FGF (R\&D Systems, MN, USA), $20 \mathrm{ng} / \mathrm{ml}$ EGF (R\&D Systems) and $5 \mu \mathrm{g} / \mathrm{ml}$ insulin (R\&D Systems). Two weeks later, sphere pictures were obtained, and the sphere formation ratios were calculated. 


\section{Animal experiments}

Subsequently, 6- to 8-week-old female BALB/c nude mice were purchased from the Experimental Animal Center of Guangdong (Foshan, Guangdong, China). To investigate the role of SGO1-AS1 in tumor metastasis and growth in vivo, luciferase-labeled SGC7901 cells overexpressing SGO1-AS1 or the control vector $\left(2 \times 10^{6}\right.$ cells per mouse) were injected into the tail vein or stomach of the $\mathrm{BALB} / \mathrm{c}$ nude mice. The luciferase signal intensity was monitored in vivo using an In Vivo Imaging System (FX PRO, Bruker, Billerica, MA, USA). Then, the mice were sacrificed, and the metastatic foci in the abdominal cavity and lung were evaluated. In addition, SGC7901 cells with SGO1-AS1 overexpression or control cells were subcutaneously injected into nude mice The mice were sacrificed 28 days after implantation, and the tumors were excised and weighed.

To confirm the inhibitory effects of SGO1-AS1 on metastasis activity via TGF $\beta$ signaling in vivo, we orthotopically implanted luciferase-labeled MKN28 cells stably expressing shSGO1-AS1 or control shRNA into the stomach of nude mice and treated the mice with saline or SB431542 $(20 \mathrm{mg} / \mathrm{kg}$ body weight, i.p.) three times per week for 3 weeks. The luciferase signal intensity was monitored in vivo by bioluminescence imaging. All animal studies were approved by the Institutional Animal Care and Use Committee of Guangzhou Medical University, and the animals were treated ethically and humanely.

\section{Statistical analysis}

A Student's t-test or chi-square test was used for the twosample comparisons. The differences among three or more groups were analyzed with a two-way analysis of variance. The overall survival curves were plotted using the Kaplan-Meier method, and the survival differences were evaluated with a log-rank test. A Cox regression was utilized to estimate the hazard ratio and 95\% confidence intervals of survival. The pairwise expression correlations were analyzed using Pearson correlation tests. P-values $<0.05$ were considered statistically significant.

\section{Results}

SG01-AS1 is downregulated in gastric carcinoma tissues and inversely associated with tumor progression

To identify GC-relevant lncRNAs, we examined the lncRNA expression profiles in five paired GC and adjacent normal mucosa tissues using a microarray. We found that 185 IncRNAs were differentially expressed in GCs compared to those in the adjacent tissues (fold change $>2$ and $P<0.05$, Fig. 1a); 13 of these lncRNAs were upregulated or downregulated by more than fourfold. We selected these lncRNAs with more than fourfold differences for qPCR expression validation in a small GC cohort (18 pairs of GC and adjacent normal tissues, Cohort 1). Among these lncRNAs, SGO1-AS1 was the most differentially expressed in $\mathrm{GC}$ relative to the normal samples (Additional file 1: Fig. S1). Furthermore, SGO1-AS1 was also downregulated in GC tissues in one publicly published dataset (GSE50710) [12] in the GEO database (Fig. 1b).

The SGO1-AS1 gene has two annotated transcripts in the Genecode database (GENCODE V23, Additional file 1: Fig. S2a). Nevertheless, the expression of the short isoform was not detected in any GC cell lines, GC tissues, or normal samples in this study (data not shown), but the long isoform was expressed to varying degrees in the GC tissues, normal samples and GC cell lines (Fig. 1c, and Additional file 1: Fig. S3a). Therefore, we focused on the long isoform of SGO1-AS1 in our further analyses. For convenience, we refer to this isoform as SGO1-AS1. In 5' and 3' RACE, SGO1-AS1 was revealed to be a 1392-nucleotide antisense transcript (Additional file 1: Fig. S2b) with a sequence that is partially complementary to SGO1 mRNAs. We examined the coding capability of SGO1-AS1 using the CodingPotential Assessment Tool (CPAT) [13] and the Coding Potential Calculator (CPC) [14]. The results showed that SGO1-AS1 has no protein-coding potential (Additional file 1: Fig. S2c). Furthermore, SGO1-AS1 was mainly located in the cytoplasm of the normal and GC cells as shown by the qRT-PCR analysis with nuclear/ cytoplasmic RNA fractionation (Additional file 1: Fig. S2d) and RNA ISH analysis (Fig. 1g).

In another cohort of 92 pairs of GC and adjacent normal tissues (Cohort 2), we further confirmed the downregulation of SGO1-AS1 in GC tissues via a qRTPCR analysis, and 63\% (58/92) of the GC cases showed more than 2-fold downregulation of SGO1-AS1 relative to the corresponding normal tissues (Fig. 1c). Moreover, decreased levels of SGO1-AS1 were correlated with clinical stage, lymph node metastasis and distant metastasis (Fig. 1d-f). This result was further confirmed by ISH analyses of 80 cases of normal gastric mucosa tissues and 95 GC tumor tissues (Cohort 3) in tissue microarrays (Fig. 1g-i, and Additional file 1: Table S5). Moreover, the survival analysis showed that low levels of SGO1-AS1 expression in GC tissues were associated with unfavorable overall survival for GC patients (log rank Chi square $=11.67, P=0.0006$, Fig. 1i). Simultaneously, the Cox proportional hazards regression analysis indicated that low SGO1-AS1 expression was an independent predictor of GC prognosis (Additional file 1: Table S6). Taken together, these results demonstrate a reverse correlation between SGO1-AS1 expression and $\mathrm{GC}$ progression. 

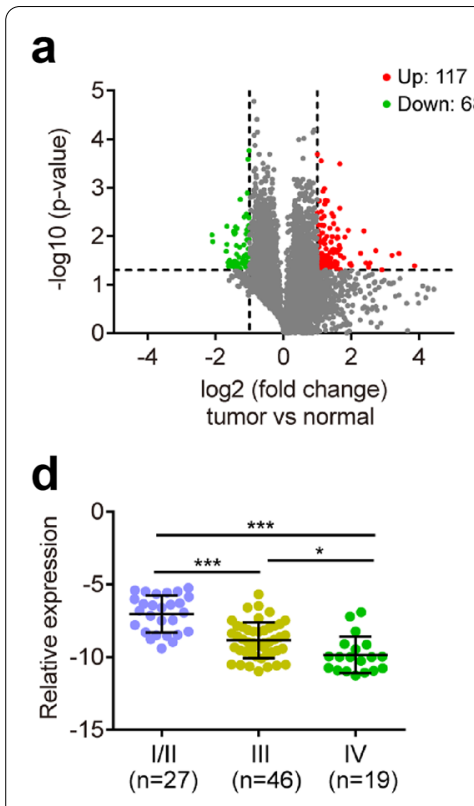

g

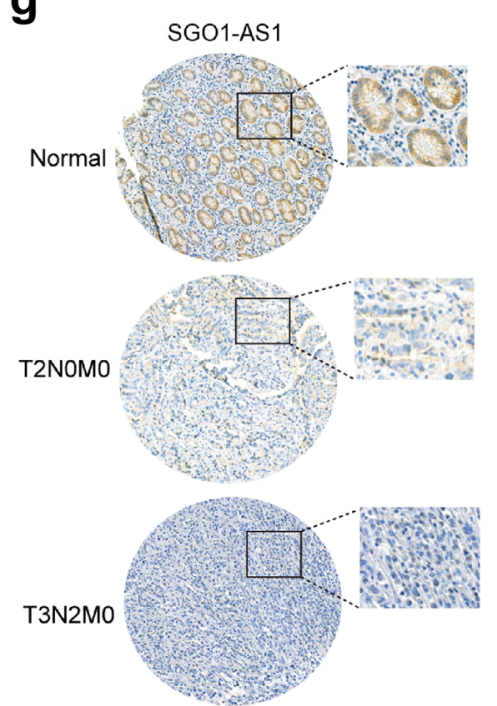

b

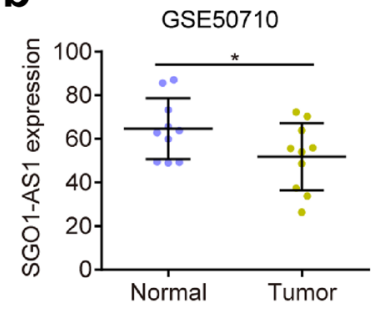

C
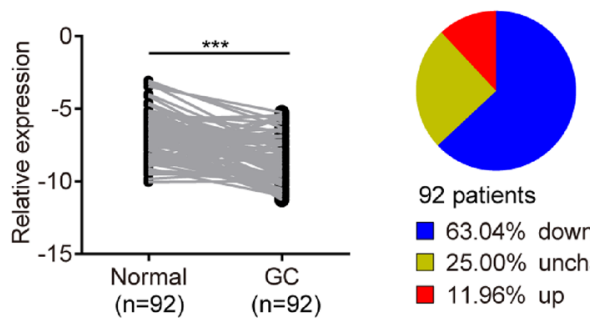

92 patients

- $63.04 \%$ down $\square 25.00 \%$ unchanged - $11.96 \%$ up

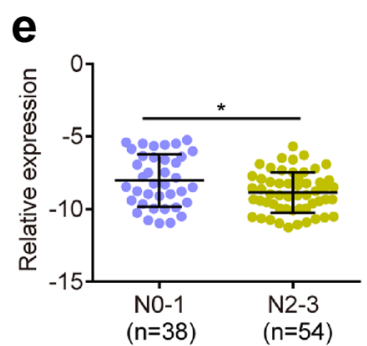

f

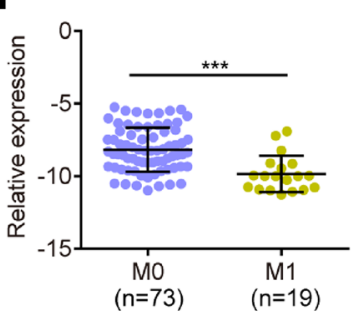

h

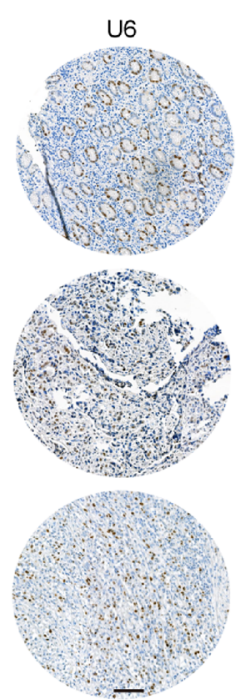

\section{j}

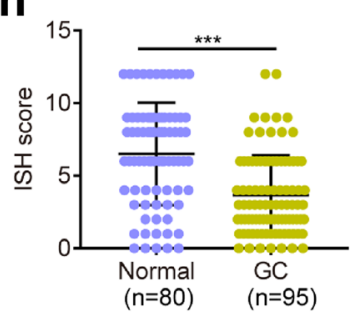

i

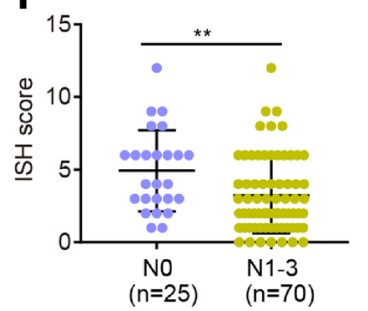

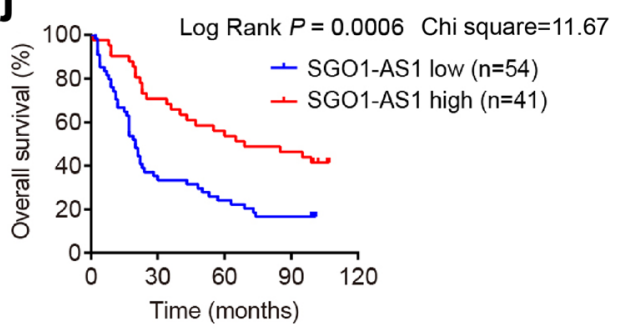

Fig. 1 SGO1-AS1 is downregulated in GC tissues and associated with GC progression. a. Volcano plots of differentially expressed IncRNAs in GCs vs. matched normal tissues shown in green and red, respectively. b. SGO1-AS1 expression levels in 10 pairs of GCs and adjacent normal tissues from the GEO dataset (GSE50710). c. Relative expression levels of SGO1-AS1 in 92 paired GC and normal tissues from Cohort 2 patients were quantified by qRT-PCR. SGO1-AS1 was downregulated (>2-fold) in 63\% (58 of 92) of the GC tissues (tumor) relative to that in the adjacent noncancerous tissues (normal). d-f. Assessment of the SGO1-AS1 expression levels in GCs according to their clinical stage (d) and status of lymph node (e) or distant metastasis (f) based on a qPCR analysis of GC. $\mathbf{g}$-i. RNA ISH analyses of SGO1-AS1 expression in 95 GC specimens and 80 normal tissues on tissue microarrays. Scale bar: $50 \mu \mathrm{m}$. j. Kaplan-Meier analyses of the overall survival of patients with GCs $(n=95)$ based on the SGO1-AS1 expression levels. The defined high and low expression levels of SGO1-AS1 were stratified according to the median expression level. Error bars indicate the standard deviation (SD). ${ }^{*} P<0.05,{ }^{* *} P<0.01,{ }^{* *} P<0.001$

\section{SG01-AS1 suppresses gastric carcinoma cell invasion and metastasis}

Given the inverse relationship between the SGO1-AS1 expression level and GC progression, we investigated whether SGO1-AS1 could affect GC cell invasion and metastasis. Therefore, we first tested the endogenous expression levels of SGO1-AS1 in gastric cell lines and found it to be expressed at low levels in the SGC7901, BGC823 and MGC803 cells and at relatively high levels in the MKN28 cells (Additional file 1: Fig. S3a). Therefore, 
SGC7901 and BGC823 cell lines were selected to stably overexpress SGO1-AS1, and MKN28 cell line was chosen to stably deplete SGO1-AS1 with effective shRNAs using a lentiviral system (Additional file 1: Fig. S3b, c). The results from the Transwell and wound-healing assays showed that stable SGO1-AS1 overexpression repressed the migration and invasion of SGC7901 and BGC823 cells (Fig. 2a, c and Additional file 1: Fig. S3d). In contrast, knockdown of SGO1-AS1 by two different shRNAs significantly enhanced the migration and invasion activities of MKN28 cells (Fig. 2b, d). Moreover, SGO1-AS1 inhibited long-term cell growth but had no significant impact on short-term growth (Additional file 1: Fig. S3e). In addition, the soft agar colony formation assays revealed that SGO1-AS1 overexpression markedly reduced the colony number and size, while silencing SGO1-AS1 had the opposite effect (Additional file 1: Fig. S3f, g).

In our subsequent in vivo study, SGC7901 cells stably expressing empty vector or SGO1-AS1 were injected into the tail vein of nude mice, and the formation of pulmonary metastases was measured. Overexpression of SGO1AS1 reduced the ability of SGC7901 cells to form lung metastases in the mice (Fig. 2e). Moreover, the survival time of the mice injected with SGC7901 cells was prolonged when SGO1-AS1 was overexpressed (Fig. 2f). In addition, SGC7901 cells with stable expression of SGO1AS1 or empty vector were injected into the corpus of the stomach of nude mice. The metastasis signals observed in the SGO1-AS1-overexpressing group were lower than those observed in the control group by bioluminescence imaging (Fig. 2g). Then, the mice were sacrificed, and the metastatic foci in the abdominal cavity were evaluated. We found that $90 \%(9 / 10)$ of the control mice, but only $40 \%$ of the mice in the SGO1-AS1-overexpressing group, had metastatic nodules in the liver (Fig. 2g). In addition, $70 \%(7 / 10)$ of the mice in the control group and only $20 \%(2 / 10)$ of the mice in the SGO1-AS1-overexpressing group had intestinal and mesenteric metastases (Fig. 2g). Subcutaneous xenografts were also established in nude mice using SGC7901 cells with stable expression of empty vector or SGO1-AS1. The overexpression of SGO1-AS1 moderately inhibited tumor growth (Additional file 1: Fig. S3h). Taken together, these findings demonstrate that SGO1-AS1 inhibits GC invasion and metastasis both in vitro and in vivo.

\section{SGO1-AS1 is associated with PTBP1}

Subsequently, we explored the molecular mechanism underlying the SGO1-AS1-induced inhibition of metastasis. SGO1-AS1 is an antisense transcript that partially overlaps the coding gene SGO1 (or SGOL1). Therefore, we examined whether SGO1-AS1 could affect the expression of the sense gene SGO1 and found that although knockdown of SGO1-AS1 moderately induced SGO1 expression, overexpression of SGO1-AS1 did not affect the expression of SGO1 (Additional file 1: Fig. S4a, b). Then, we identified potential SGO1-AS1-interacting proteins. We performed RNA pull-down assays in vitro with biotinylated SGO1-AS1, followed by SDS-PAGE electrophoresis, and an overtly differential band at approximately $60 \mathrm{kD}$ in the sense lane was selected for mass spectrum analyses (Fig. 3a). Our results revealed several potential proteins that were pulled down with SGO1AS1 RNA, and of these proteins, PTBP1 received the highest score (Additional file 1: Table S7). Biotin-labeled RNA pulldown followed by Western blotting analysis confirmed that PTBP1 and G3BP2 are SGO1-AS1-binding proteins (Fig. 3b). Furthermore, RIP followed by qRTPCR assays showed that antibodies against either PTBP1 or G3BP2 could significantly enrich for SGO1-AS1 compared to the controls (Fig. 3c).

PTBP1 is a member of the heterogeneous nuclear ribonucleoprotein (hnRNP) family and a critical regulator of mRNA splicing [15, 16], RNA stability [17], transportation [18], localization [19] and translation [20, 21]. PTBP1 has been shown to be involved in tumorigenesis, although its effects on malignancy appear to be cell-type dependent [16, 22, 23]. We focused on PTBP1 as an interacting partner of SGO1-AS1 for further investigation. To determine the region of SGO1AS1 to which PTBP1 binds, we prepared a series of biotin-labeled SGO1-AS1 probes with deletion mutants and performed an in vivo RNA pull-down experiment. We found that the 1-415 nt fragment of SGO1-AS1 was sufficient to bind PTBP1 (Fig. 3d). Considering that PTBP1 binds pyrimidine-rich sequences (UCUUC), we

\footnotetext{
(See figure on next page.)

Fig. 2 SGO1-AS1 suppresses the invasive and metastatic activity of GC cells in vitro and in vivo. a-b. Transwell assay measuring the invasion of SGC7901 and BGC823 cells stably expressing SGO1-AS1 (a), MKN28 cells stably silencing SGO1-AS1 (b) and their respective control cells. c-d. Wound healing assay measuring the migratory ability of the indicated GC cells. a-d Scale bars, $150 \mu \mathrm{m}$. Error bars, SD from three independent experiments performed in triplicate. ${ }^{*} P<0.05,{ }^{* *} P<0.01,{ }^{* * *} P<0.001$. e. SGC7901 cells stably expressing SGO1-AS1 or vector were intravenously injected into the tail vein of nude mice. Representative bioluminescent images and H\&E-stained lung sections of the mice are shown. The number of metastatic foci was quantified. Scale bar, $200 \mu \mathrm{m}$. Error bars represent SD ( $n=5$ mice/group). Arrows or circles indicate metastatic nodules. ${ }^{*} P<0.01$. f. Kaplan-Meier survival curve of mice in a parallel experiment. g. SGO1-AS1-overexpressing SGC7901 cells and control cells were orthotopically injected into the stomach of nude mice (10 mice per group). The mice were sacrificed 45 days later, and the tumor nodules in the abdominal cavity were examined. Representative IVIS luciferase in vivo images and bright views of livers and intestines isolated from the mice are shown. The bioluminescence signal and number of mice with metastasis were quantified. Arrows or circles indicate metastatic nodules. ${ }^{* *} P<0.01$
} 


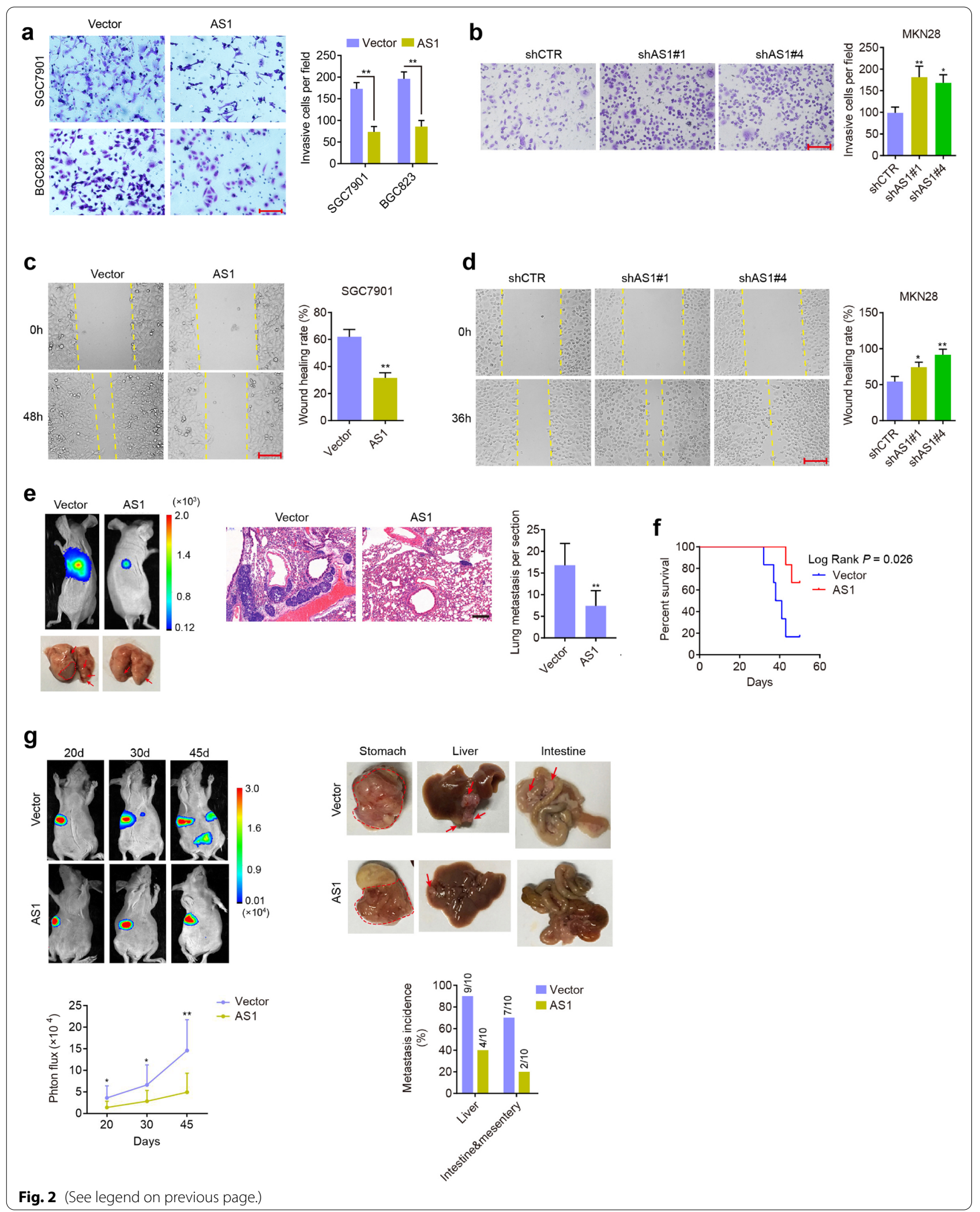



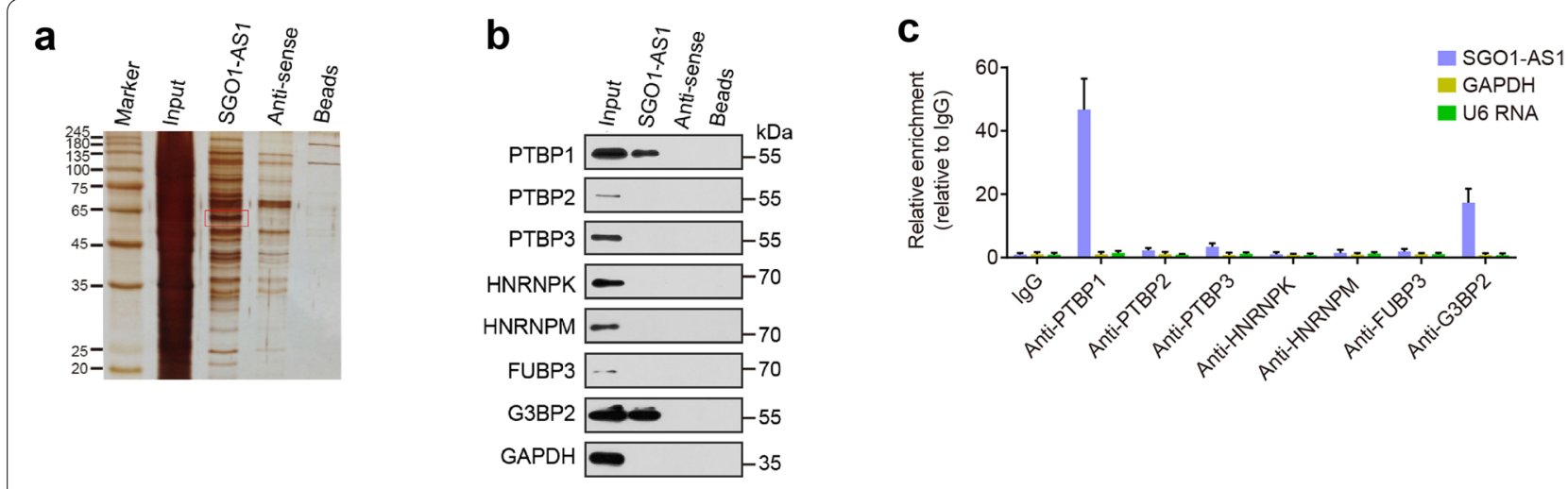

d
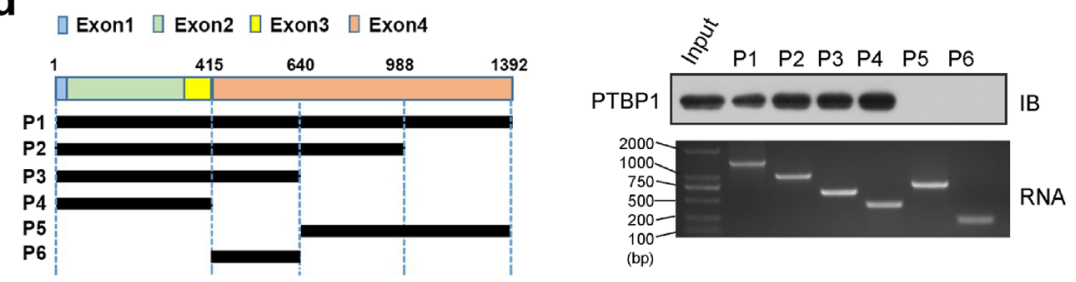

e

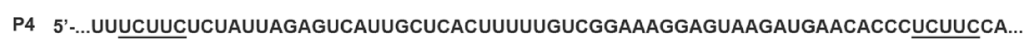
P4 mut 5 ;....UUACACCUCUAUUAGAGUCAUUGCUCACUUUUUGUCGGAAAGGAGUAAGAUGAACACCCCCAACCA...
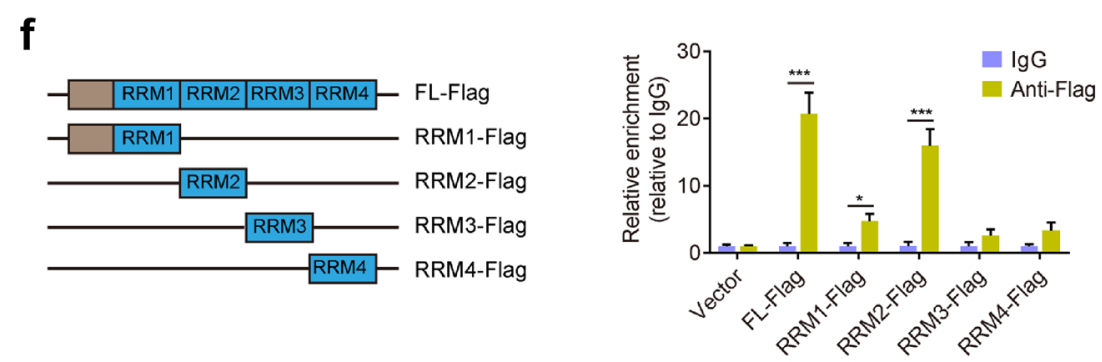

Fig. 3 SGO1-AS1 RNA interacts with the PTBP1 protein. a. Identification of SGO1-AS1-associated proteins by an RNA pulldown assay. The proteins pulled down by SGO1-AS1 or the antisense RNA of SGO1-AS1 incubated with SGC7901 cell extracts were resolved by SDS-PAGE and subjected to silver staining. A specific band was identified in the SGO1-AS1 group and is marked with a red box. $\mathbf{b}$. Western blot analysis validation of the biotin-labeled RNA pulldown assay using sense or antisense probes in SGC7901 cells. GAPDH was used as a negative control. c. RIP-qPCR detection of the indicated RNAs retrieved by specific antibodies in MKN28 cells. The fold enrichment of SGO1-AS1 relative to lgG was determined by qRT-PCR. U6 RNA and GAPDH were used as negative controls. $\mathbf{d}$. Serial deletions of SGO1-AS1 were used in the RNA pulldown assays to identify the core regions of SGO1-AS1 required for a physical interaction with PTBP1. Left panel: graphic illustration of the SGO1-AS1 probes. e. RNA pulldown assay was performed using biotin-labeled 1-415 nt (P4) and PTBP1-binding site mutated RNA (P4 mut) of SGO1-AS1, followed by a Western blot analysis. f. RIP assays were performed using an anti-Flag antibody in HEK293T cells transfected with Flag-tagged PTBP1 or its deletion mutants. qRT-PCR was used to measure the enrichment of SGO1-AS1. Error bars represent SD. ${ }^{*} P<0.05,{ }^{* *} P<0.001$

identified the accurate binding site of PTBP1 in SGO1AS1 (258-319 nt) via RNA pulldown assays (Fig. 3e). PTBP1 contains four RRM domains for binding RNA [24]. To investigate which domain of PTBP1 accounts for its interaction with SGO1-AS1, we performed RIP assays using a series of Flag-tagged PTBP1 deletion mutants and found that the RRM2 domain of PTBP1 had the strongest association with SGO1-AS1 (Fig. 3f). In addition, we observed that neither overexpression nor knockdown of SGO1-AS1 could influence the expression levels of PTBP1 (Fig. 4m). 
SGO1-AS1 regulates TGFB1/2 mRNA stability by interacting with PTBP1

PTBP1 is an RNA-binding protein (RBP) that plays a role in mRNA metabolism by binding target mRNAs [20]. We hypothesized that the association between SGO1-AS1 and PTBP1 may influence the effects of PTBP1 on its target mRNAs. We first evaluated the expression levels of PTBP1 in gastric cell lines and found it to be expressed at high levels in almost all GC cell lines (Additional file 1: Fig. S5a). Then, we generated a stable PTBP1-knockout MKN28 cell line using CRISPR-Cas9 technology (Fig. 4a, e) and performed an RNA-seq analysis. The comparison of the PTBP1-knockout and control cells revealed that 393 mRNAs were upregulated and 348 mRNAs were downregulated (Fig. 4b). The Kyoto Encyclopedia of Genes and Genomes (KEGG) analysis revealed that the downregulated genes were apparently enriched in TGF $\beta$ signaling and pathways regulating stem cells, the extracellular matrix (ECM) and focal adhesion (Fig. 4c). The downregulated genes in the PTBP1-knockout MKN28 cells involved in the TGF $\beta$ pathway were further validated at the mRNA and protein levels (Fig. 4d, e). A similar result was observed in the SGC7901 cells transfected with PTBP1 shRNA (Additional file 1: Fig. S5b). Interestingly, based on the SGO1-AS1 levels, we performed a gene set enrichment analysis (GSEA) using The Cancer Genome Atlas (TCGA: https://cancergenome.nih.gov) stomach adenocarcinoma RNA-seq dataset and demonstrated that TGF $\beta 1$ target genes were significantly enriched in GC patients with low SGO1-AS1 expression (Fig. 4f), implying a role of this lncRNA in regulating TGF $\beta$ signaling.

Given that PTBP1 is an RNA binding protein [20], we performed RIP-seq experiments using an anti-PTBP1 antibody or rabbit immunoglobulin $G$ to reveal the PTBP1-bound RNAs. We identified 5186 transcripts potentially bound by PTBP1 and 173 transcripts that overlapped between the PTBP1-bound mRNAs and differentially expressed genes following PTBP1 knockout; three of these transcripts (TGFB1, TGFB2 and ID2) were related to the TGF $\beta$ pathway (Fig. 4g). The interaction between PTBP1 and TGFB1, TGFB2 or ID2 mRNA was further confirmed by RIP followed by qPCR (Fig. 4h and Additional file 1: Fig. S5c). Importantly, SGO1-AS1 overexpression resulted in a large increase in the association between SGO1-AS1 and PTBP1, while the TGFB1/2 mRNA binding of PTBP1 was markedly decreased in the SGC7901 cells (Fig. 4i). In contrast, the association between TGFB1/2 mRNA and PTBP1 was increased following the knockdown of SGO1-AS1 in the MKN28 cells (Fig. 4j). However, the regulation of SGO1-AS1 expression did not influence the association between the PTBP1 protein and ID2 mRNA (Additional file 1: Fig. S5d). Furthermore, we found that the TGFB1/2 mRNA mainly bound the RRM2 domain of PTBP1 (Additional file 1: Fig. S5e), which is the region bound by SGO1-AS1 (Fig. 3f). Altogether, these results suggest that SGO1-AS1 competes with TGFB1/2 mRNA to bind PTBP1.

Since our above results indicate that depletion of PTBP1 reduces the TGFB mRNA levels, we investigated whether PTBP1 and SGO1-AS1 may influence TGFB mRNA stability. Actinomycin D (ACD) was used to block de novo transcription in PTBP1-knockout and control cells infected with SGO1-AS1 shRNA. As expected, the depletion of PTBP1 increased TGFB mRNA degradation following ACD treatment in MKN28 cells. However, silencing SGO1-AS1 stabilized TGFB mRNAs but had no significant effect on TGFB mRNA stability under conditions in which PTBP1 was depleted (Fig. 4k). Furthermore, the forced expression of SGO1-AS1 facilitated TGFB1/2 mRNA degradation, and the co-overexpression of PTBP1 abrogated this effect, although the overexpression of PTBP1 alone failed to influence TGFB mRNA stability (Fig. 4l), indicating that PTBP1 is necessary for the SGO1-AS1-mediated TGFB mRNA decay. Consistently, knockdown of SGO1-AS1 increased the TGF $\beta 1 / 2$ protein level, which was offset following PTBP1 knockout (Fig. 4m). Conversely, SGO1-AS1 overexpression remarkably downregulated TGF $\beta$ protein expression, and this downregulation was reversed by the coexpression of

\footnotetext{
(See figure on next page.)

Fig. 4 SGO1-AS1 promotes TGFB1/2 mRNA decay by interacting with PTBP1. a. PTBP1-knockout (KO) MKN28 cells were produced using the CRISPR/Cas9 system. Disruption of the PTBP1 locus. b. Volcano plots showing the differentially expressed genes in the PTBP1-KO vs. control cells. c. KEGG analysis of the downregulated genes in the PTBP1-KO vs. control cells. d-e. qRT-PCR and Western blot analysis were used to validate the expression of genes involved in the TGF $\beta$ pathway in the indicated cells. f. GSEA results plotted to illustrate the correlation between the expression of SGO1-AS1 and TGF $\beta$ target genes in the TCGA stomach adenocarcinoma RNA-seq dataset. g. Overlay of differentially expressed genes following the PTBP1 knockout and PTBP1-binding target mRNAs. h. PTBP1 RIP assay was performed to analyze the interactions between the PTBP1 protein and TGFB1/2 or ID2 mRNA in MKN28 cells. The relative fold enrichment of these mRNAs compared to lgG was determined by qRT-PCR. SMAD5 and GAPDH served as negative controls. $\mathbf{i}$-j. The enrichment of SGO1-AS1 RNA and TGFB1/2 mRNA in PTBP1 immunoprecipitants was detected by a RIP-qPCR assay in SGC7901 cells with SGO1-AS1 overexpression (i) and MKN28 cells with SGO1-AS1 knockdown (j), respectively. $\mathbf{k}-\mathrm{I}$. TGFB1/2 mRNA stability assessment in the indicated cells treated with actinomycin D $(5 \mu \mathrm{g} / \mathrm{mL})$ for $2,4,8$ and $16 \mathrm{~h}$. TGFB1/2 mRNA abundance relative to GAPDH quantified by qRT-PCR ( $n=3$ independent experiments). $\mathbf{m}$. Western blotting analysis of the TGF $\beta 1 / 2$ and PTBP1 protein levels in the indicated cells. n. TGFB1/2 mRNA stability assessment in control and PTBP1-KO MKN28 cells transfected with siAGO2 or control siRNA. Error bars represent SDs. ${ }^{*} P<0.05,{ }^{* *} P<0.01$, ${ }^{* * *} P<0.001$
} 

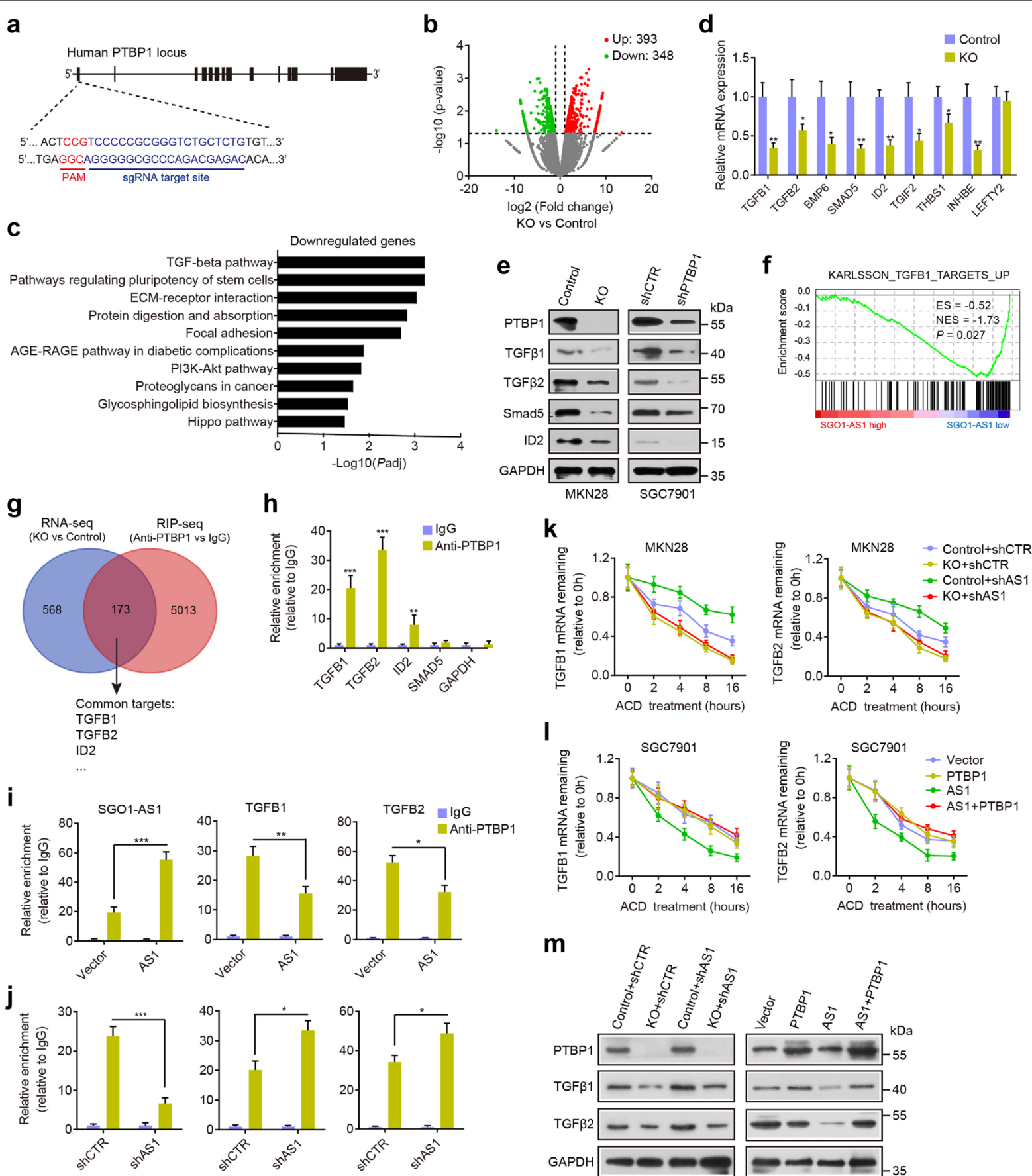

n
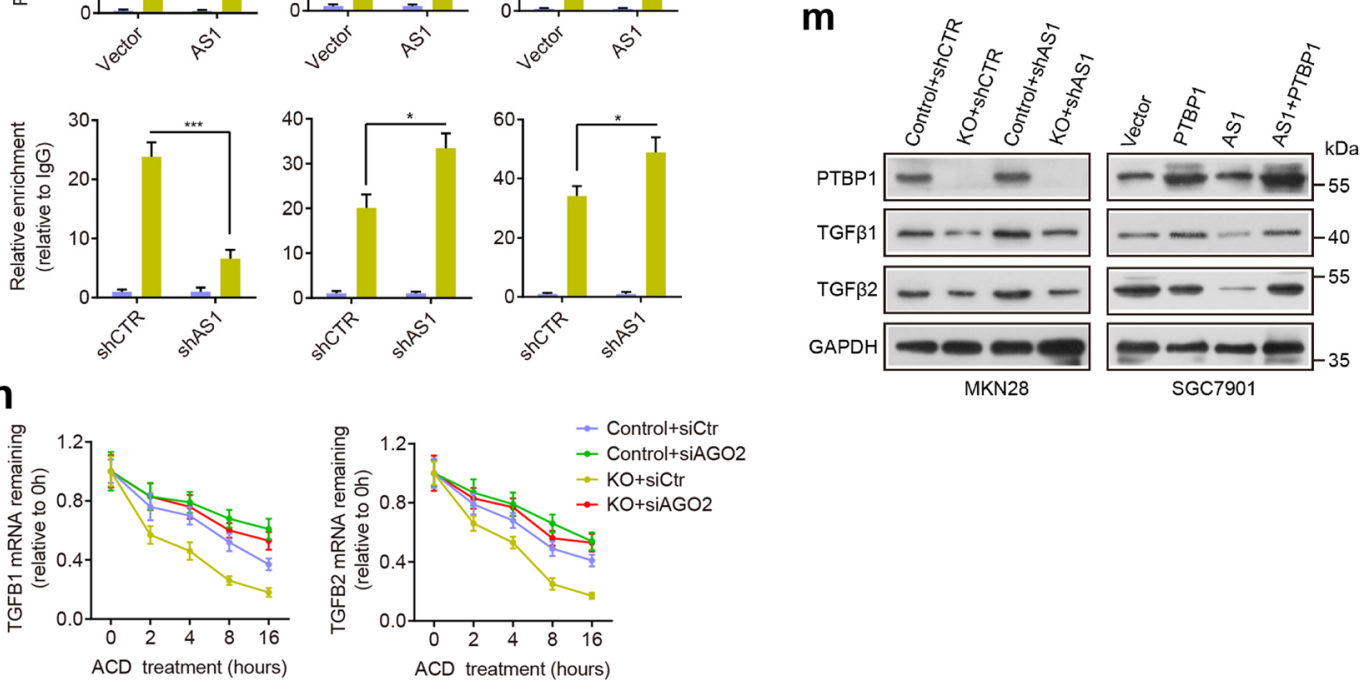

Fig. 4 (See legend on previous page.) 
PTBP1 (Fig. 4m). Moreover, the xenograft tumors generated by SGO1-AS1-overexpressing SGC7901 cells featured lower TGFB1/2 expression than the control tumors (Additional file 1: Fig. S5f, g). Overall, these results demonstrate that SGO1-AS1 inhibits TGFB1/2 mRNA expression via a PTBP1-mediated mechanism.

Next, we explored the mechanism by which PTBP1 promotes the stability of TGFB $1 / 2$ mRNA. Numerous reports indicate that the RNA-induced silencing complex (RISC) mediates mRNA decay $[25,26]$, and we hypothesized that this mechanism may account for the PTBP1mediated TGFB stability. To address this, we knocked down AGO2, a major element of RISC, with siRNAs and found that knockdown of AGO2 increased TGFB1/2 expression levels in PTBP1-KO cells (Additional file 1: Fig. S5h). Moreover, the TGFB mRNA degradation was decreased in PTBP1-KO cells transfected with siAGO2 compared with the control (Fig. 4n). We next determined whether AGO2 complexes with TGFB1/2 mRNA. RIP assays showed that AGO2 could be significantly enriched in TGFB mRNA (Additional file 1: Fig. S5i). Importantly, depletion of PTBP1 significantly enhanced but silencing of SGO1-AS1 reduced the enrichment of AGO2 in TGFB1/2 mRNA (Additional file 1: Fig. S5h). Together, these results indicate that PTBP1 prevents AGO2 recruitment of TGFB mRNA to form RISC system, causing a decrease in decay of TGFB mRNA.

\section{SGO1-AS1 impedes TGF $\beta$ signaling, the EMT and stemness} As SGO1-AS1 reduces TGF $\beta$ production, we evaluated whether SGO1-AS1 may affect TGF $\beta$ downstream signaling. As expected, silencing SGO1-AS1 enhanced the phosphorylation of SMADs and induced the transcriptional activity of SBE4 (SMAD-binding element) in control cells but showed little effect in the PTBP1-deficient cells, and the activation of TGF $\beta$ signaling by SGO1AS1 knockdown was reversed by the treatment with the TGF $\beta$ type I receptor (T $\beta R I$ ) inhibitor SB431542 (Fig. 5a, b). These results indicate that SGO1-AS1 acts as a potent antagonist of TGF $\beta / S M A D$ signaling in a PTBP1dependent manner.

TGF $\beta$ is a major inducer of the EMT and stimulates stemness, invasion and metastasis in cancer cells $[27,28]$. Therefore, we examined whether silencing SGO1-AS1 could promote the ability of GC cells to undergo the EMT. As shown in Fig. 5c and Additional file 1: Fig. S6a, knockdown of SGO1-AS1 in MKN28 cells reduced the epithelial features but increased the mesenchymal features as evidenced by the reduced expression of an epithelial marker (E-cadherin) but increased levels of mesenchymal markers (Vimentin and N-cadherin) and the elongation of cell bodies. We also examined the levels of the EMT-inducing transcription factors (EMT-TFs) ZEB1, ZEB2, TWIST1, SNAI and SLUG and showed that the expression levels of ZEB1, SNAI and SLUG were increased upon SGO1-AS1 knockdown (Fig. 5e). Moreover, the SGO1-AS1 knockdown-induced mesenchymal-like features were reversed by PTBP1 knockout or SB431542 treatment (Fig. 5c, e and Additional file 1: Fig. S6a). In contrast, SGO1-AS1 overexpression in SGC7901 cells dampened the EMT process, which was rescued by the treatment with the recombinant TGF $\beta 1$ protein (Fig. 5d, e and Additional file 1: Fig. S6a). Consistently, the invasive activity was significantly decreased in the SGO1-AS1-deleted MKN28 cells upon PTBP1 loss or SB431542 treatment but was rescued in the SGO1-AS1-overexpressing SGC7901 cells with the addition of recombinant TGF $\beta 1$ (Fig. $5 \mathrm{f}$ and Additional file 1: Fig. S6b). To confirm the inhibitory effects of SGO1-AS1 on metastatic activity through TGF $\beta$ signaling in vivo, we orthotopically implanted SGO1-AS1 knockdown cells into nude mice and treated them with SB431542 (three times per week). Consistent with the overexpression study (Fig. 2g), the metastasis signals found in the SGO1AS1 knockdown group were higher than the signals in the control group, while the SB431542 treatment exhibited a significant reduction in metastases in the mice harboring SGO1-AS1 knockdown cells (Fig. 5j).

Then, we examined the effects of SGO1-AS1 on stemness. SGO1-AS1 knockdown increased tumor spheroid formation and the ALDH1+ population, whereas loss of PTBP1 or treatment with SB431542 significantly abolished these effects (Fig. $5 \mathrm{~g}, \mathrm{~h}$ and Additional file 1: Fig. S6c). In contrast, SGO1-AS1 overexpression reduced the stemness features in SGC7901 cells, and the addition of TGF $\beta 1$ induced stemness features in the SGO1-AS1overexpressing cells. Moreover, the in vivo limiting dilution tumorigenicity assays showed that the SGO1-AS1

(See figure on next page.)

Fig. 5 SGO1-AS1 impedes TGF $\beta$ signaling, the EMT and stemness. a-b. SGO1-AS1 knockdown increased SMAD2/3 phosphorylation and SBE4 transcriptional activity, and this effect was reversed by PTBP1 knockout or SB431542 (10 $\mu \mathrm{M}, 24 \mathrm{~h})$ as shown by Western blot (a) and luciferase reporter assays of the SBE4 promoter (b). c-d. EMT markers were measured in the indicated cells by a Western blot analysis. e. qRT-PCR analysis of EMT-TF expression in the indicated cells. $\mathbf{f}$-h. Cell invasion ability (f), tumorsphere formation efficiency $\mathbf{( g )}$ ) and rates of ALDH1-positive cells (h) in the indicated cells. i. Limiting dilution xenograft formation of SGC7901 cells stably expressing SGO1-AS1 or vector ( $n=5$ mice per group). j. MKN28 cells with SGO1-AS1 knockdown or control were orthotopically injected into the stomach of nude mice, and the mice were treated with PBS or SB431542 (20 mg $/ \mathrm{kg}$ body weight, i.p.) three times per week for 3 weeks ( $n=6$ mice per group). Tumor progression was monitored by luciferase signal intensity using an In Vivo Imaging System. Error bars indicate SDs. ${ }^{*} P<0.05,{ }^{*} P<0.01,{ }^{* *} P<0.001$ 


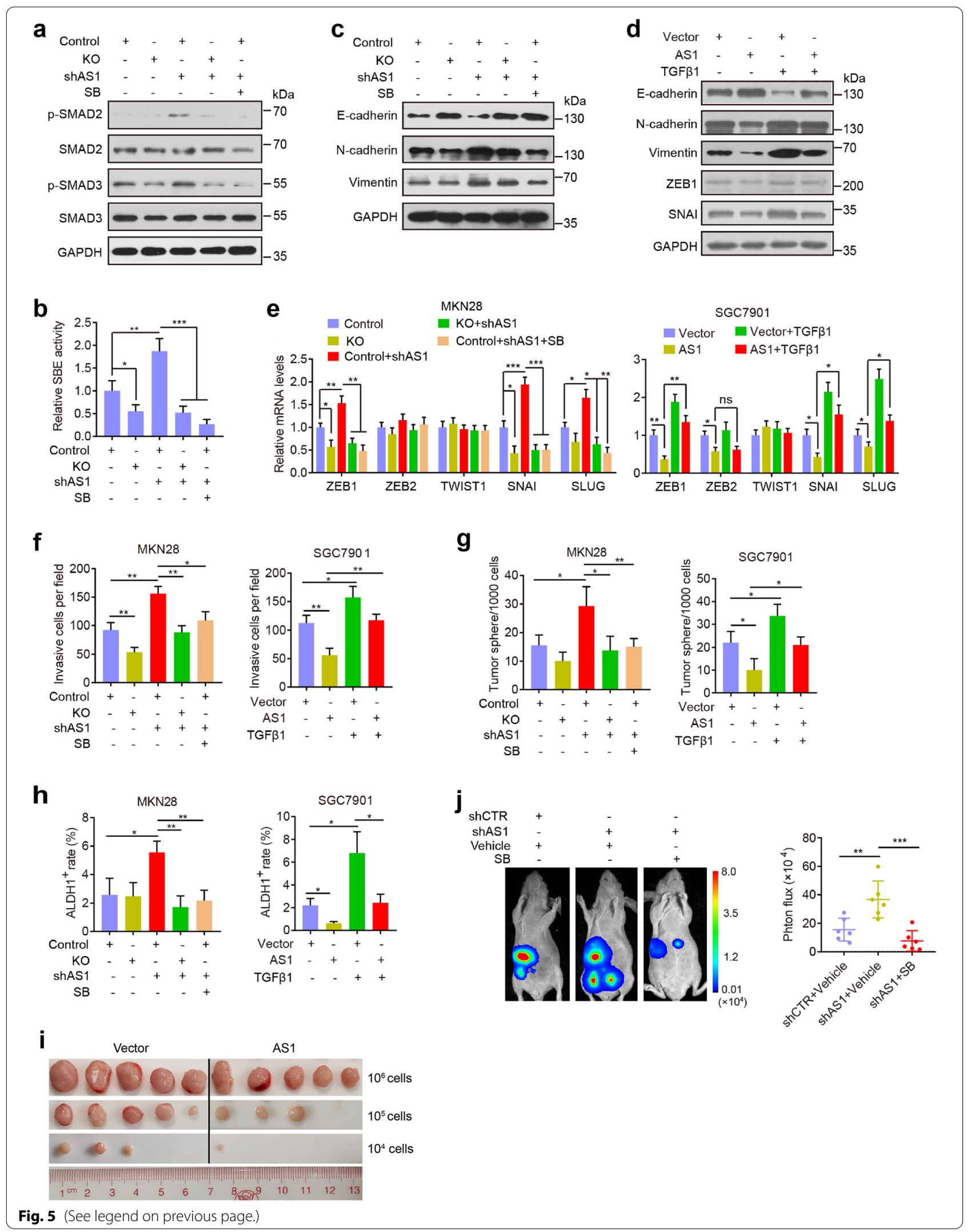


overexpression also inhibited the tumorigenic potential of the SGC7901 cells in the nude mice (Fig. 5i). Collectively, these data indicate that SGO1-AS1 attenuates the EMT, stemness and metastasis via PTBP1-mediated TGF $\beta$ signaling.

\section{SG01-AS1 reduces TGF $\beta$ autocrine signaling}

Because our results showed that SGO1-AS1 reduced TGF $\beta$ expression and its downstream signaling, we reasoned that SGO1-AS1 might influence the secretion of these cytokines to interrupt the tumor microenvironment. Indeed, SGO1-AS1 knockdown induced, but SGO1-AS1 overexpression inhibited, the secretion of these cytokines as observed in enzyme-linked immunosorbent assays (ELISAs) (Fig. 6a, b). To confirm that SGO1-AS1 modulates TGF $\beta$ autocrine signaling, we collected conditioned medium from SGO1-AS1knockdown MKN28 cells or SGO1-AS1-overexpressing SGC7901 cells, transferred the conditioned medium to HEK293T cells and performed SBE4 transcription assays. The results showed that the conditioned medium collected from the SGO1-AS1-silenced cells activated SBE4 transcription (Fig. 6c). In contrast, SBE4 transcription in HEK293T cells treated with conditioned medium collected from SGO1-AS1-overexpressing cells was downregulated compared to that in the control cells. Next, we explored the invasive phenotype of GC cells incubated with conditioned medium. As expected, MKN28 cells incubated with conditioned medium collected from SGO1-AS1-silenced MKN28 cells exhibited higher migration and invasion capacities than the cells incubated with conditioned medium collected from control cells, whereas SGC7901 cells incubated with conditioned medium collected from SGO1-AS1-overexpressing SGC7901 cells had reduced migration and invasion abilities compared to the cells incubated with conditioned medium collected from control SGC7901 cells (Fig. 6d, e). These data indicate that SGO1-AS1 reduces the autocrine activity of TGF $\beta$ cytokines.

\section{TGF $\beta$ represses SGO1-AS1 transcription via ZEB1}

Having found that SGO1-AS1 antagonizes TGF $\beta$ signaling by promoting TGFB mRNA degradation, we investigated the response of SGO1-AS1 to TGF $\beta$. We treated SGC7901 cells with TGF $\beta 1$ and found that SGO1AS1 expression was inhibited, but well-known TGF $\beta$ targets (SNAI and ZEB1) $[29,30]$ were induced in a dose-dependent manner (Fig. 7a). SGO1-AS1 downregulation by the TGF $\beta$ treatment was confirmed in two other GC cell lines (Fig. 7b). Then, we exposed the cells to the TGFBRI inhibitor SB431542 and found that the SB431542 treatment induced SGO1-AS1 expression, which was accompanied by the downregulation of
SNAI and ZEB1 (Fig. 7c). We carried out a bioinformatics analysis using JASPAR (http://jaspar.genereg.net/) to identify potential regulatory transcription factors. The bioinformatics analysis revealed four ZEB1 binding motifs at -222 to -214 (site A), -731 to -721 (site B), -822 to -814 (site C) and -947 to -940 (site D) inside the SGO1-AS1 promoter (Fig. 7d). ZEB1 is a downstream target gene of TGF $\beta$ and has been reported to be a master regulator of the EMT and cancer metastasis [31]. We evaluated whether the downregulation effects of TGF $\beta$ on SGO1-AS1 occur via ZEB1. The luciferase reporter assay showed that the luciferase activity of the reporter constructs containing $1 \mathrm{~kb}$ of the wild-type SGO1-AS1 promoter was decreased by approximately 2 -fold in the TGF $\beta$-treated cells relative to that in the control cells, and the ZEB1 binding site mutation on the SGO1-AS1 promoter abolished this effect (Fig. 7e). Then, we knocked down ZEB1 in GC cells treated with TGF $\beta 1$ to study its effects on the SGO1-AS1 levels and found that depletion of ZEB1 completely reversed the repressive effects of TGF $\beta 1$ on SGO1-AS1 expression (Fig. 7f). Using ChIP, we discovered significant enrichment in the binding of ZEB1 to the promoter region of SGO1-AS1 in the cells treated with TGF $\beta 1$ relative to the control cells (Fig. $7 \mathrm{~g}$ ). Taken together, our data indicate that TGF $\beta$ downregulates SGO1-AS1 by inducing ZEB1.

\section{SG01-AS1 downregulation is correlated with high expression of TGFB1/2 and ZEB 1 in gastric carcinoma specimens}

We sought to determine whether our findings could be extended to patients with gastric carcinoma. The expression levels of TGFB1, TGFB2, ZEB1 and PTBP1 were detected by qRT-PCR in 92 pairs of gastric carcinoma specimens and adjacent normal tissues (Cohort 2 ). The expression levels of these four genes were significantly increased in the tumors compared to those in the normal tissues, and $51.1 \%$ (47/92), 58.7\% (54/92) $39.1 \%(36 / 92)$, and $52.2 \%(48 / 92)$ of the GC samples showed more than a 2-fold upregulation of TGFB1, TGFB2, ZEB1 and PTBP1, respectively (Fig. 8a-c and Additional file 1: Fig. S7a). Moreover, high levels of TGFB1/2 and ZEB1 were significantly associated with lymphatic invasion and advanced tumor stage (Fig. 8d-f). GCs with metastasis expressed higher levels of TGFB1/2, ZEB1 and PTBP1 than those without metastasis (Fig. 8d-f and Additional file 1: Fig. S7b). Importantly, TGFB1/2 and ZEB1 were inversely correlated with SGO1-AS1, whereas TGFB1/2 was positively associated with ZEB1 in the GC tissues (Fig. 8g), further confirming the SGO1-AS1-TGFB-ZEB1 regulatory axis in GC. In addition, there was no significant correlation between PTBP1 and SGO1-AS1 expression 

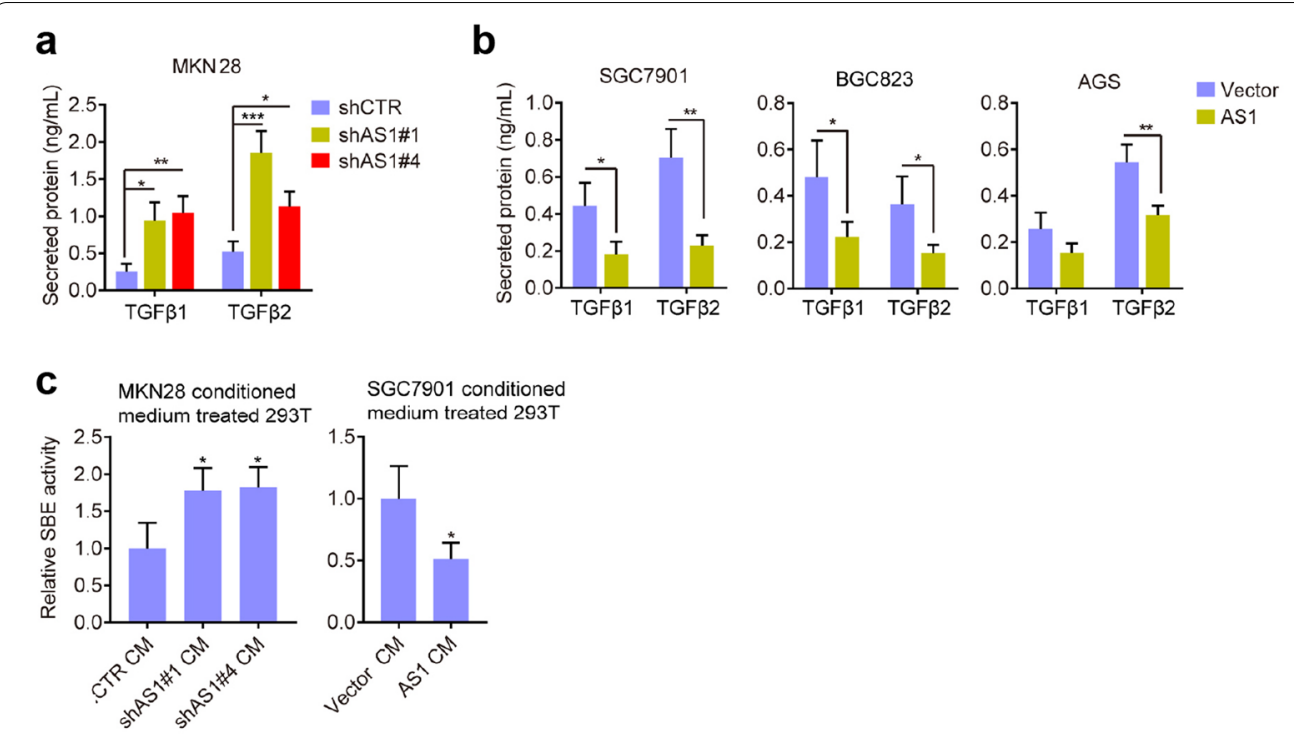

d
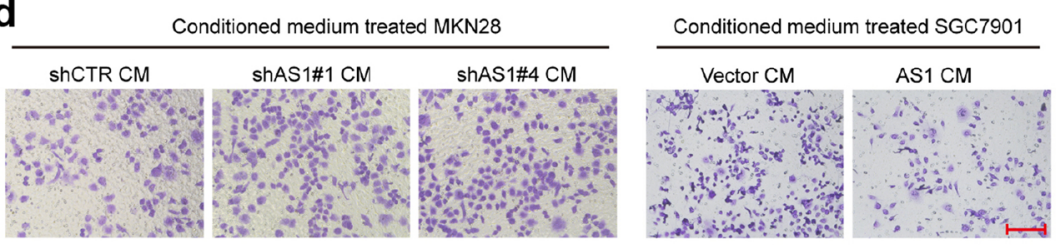

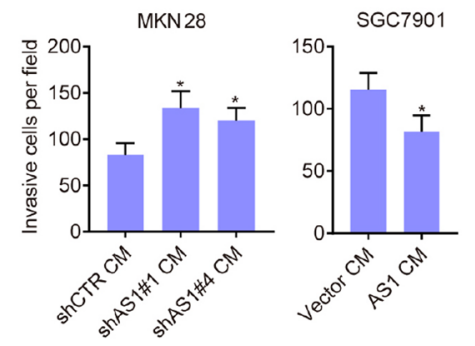

e

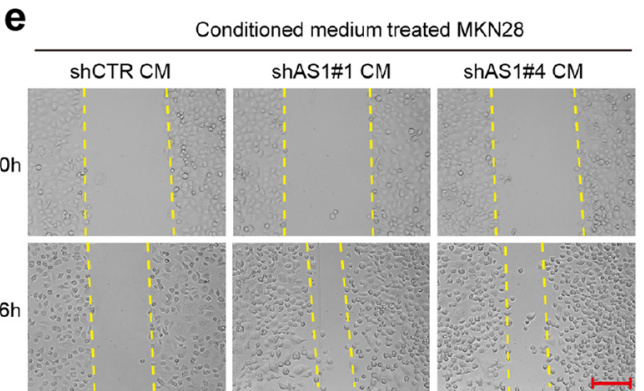

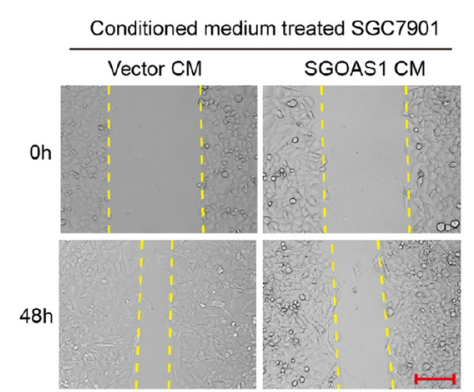

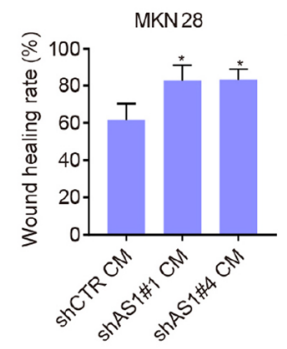

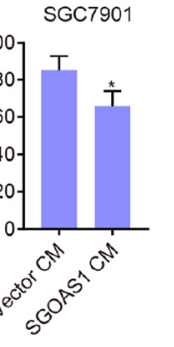

Fig. 6 SGO1-AS1 decreases TGF $\beta$ autocrine signaling. a-b. ELISA analysis of the TGF $\beta 1 / 2$ protein levels in conditioned medium from cells with SGO1-AS1 knockdown (a) or overexpression (b). c. Conditioned medium from SGO1-AS1-silenced MKN28 cells enhanced SBE4 promoter luciferase activity in HEK293T cells. In contrast, SBE4 transcription in HEK293T cells treated with conditioned medium collected from the SGO1-AS1-overexpressing SGC7901 cells was downregulated compared to that in the cells treated with medium collected from the control cells. d. The cell invasion ability of MKN28 cells increased when treated with conditioned medium from SGO1-AS1-silenced MKN28 cells, but that of SGC7901 cells was decreased following the treatment with conditioned medium from SGO1-AS1-overexpressing SGC7901 cells. e. The migratory ability of MKN28 cells was increased following the treatment with conditioned medium from SGO1-AS1-silenced MKN28 cells, while that of SGC7901 cells was decreased following the treatment with conditioned medium from SGO1-AS1-overexpressing SGC7901 cells. Scale bar, $150 \mu \mathrm{m}$. All error bars indicate SDs. ${ }^{*} P<0.05,{ }^{* *} P<0.01,{ }^{* * *} P<0.001$

or between TGFB1 and TGFB2 expression (Fig. $8 \mathrm{~g}$ and Additional file 1: Fig. S7c). We also observed that PTBP1 was significantly associated with TGFB1 expression (Additional file 1: Fig. S7c).

\section{Discussion}

Most patients with GC die from metastatic disease, but knowledge regarding the mechanisms of metastasis in gastric tumors is limited [2]. In this study, we 
identified a metastasis-suppressive lncRNA, i.e., SGO1AS1, which is decreased in progressed gastric cancer and inversely correlated with gastric tumor metastasis. We further revealed that SGO1-AS1 interacts with the protein PTBP1, and their interaction competitively reduces TGFB1/2 mRNA binding to PTBP1. In turn, the decreased binding of TGFB1/2 mRNA to PTBP1 leads to a reduction in TGFB1/2 mRNA stability and reduced TGF $\beta$ production, thus preventing the EMT and metastasis. In addition, TGF $\beta$ represses SGO1-AS1 transcription by inducing ZEB1. Thus, SGO1-AS1 and TGF $\beta$ form a double-negative feedback loop via ZEB1 to regulate the EMT and metastasis (Fig. 8h).

LncRNAs often exert their effects through the proteins with which they interact [32]. Here, we identified PTBP1 as an SGO1-AS1-interacting protein. PTBP1 has been shown to be involved in tumorigenesis by regulating alternative splicing [33, 34], controlling mRNA stability $[17,35]$ and determining mRNA localization [19]. For example, PTBP1 enhances the PKM2 isoform and reduces the PKM1 isoform by controlling PKM alternative splicing, which promotes aerobic glycolysis and provides a selective advantage for tumor formation [16, 36]. PTBP1 mediates MCL1 mRNA stability and regulates cellular apoptosis induced by antitubulin chemotherapeutics [23]. Notably, several lncRNAs have been reported to be associated with PTBP1 [17, 37, 38]. The hypoxia-induced lncRNA LUCAT1 interacts with PTBP1 in CRC cells, facilitating the association between a set of DNA damage-related genes and PTBP1 and resulting in altered alternative splicing of these genes, thereby conferring resistance to chemotherapeutic drugs in CRC cells [39]. The lncRNA MEG3 can recruit PTBP1 to regulate small heterodimer partner mRNA stability and cholestatic liver injury [17]. Recruiting PTBP1 to target mRNAs appears to be a common mechanism among these lncRNAs. However, we found that the interaction between SGO1-AS1 and PTBP1 reduces the enrichment of this protein in TGFB1/2 mRNA to facilitate their decay. In addition to PTBP1, it is possible that SGO1-AS1 might bind other proteins, such as G3BP2, to regulate $\mathrm{GC}$ metastasis as G3BP2 was found to be an
SGO1-AS1-interacting protein in the mass spectrum analyses and verification analyses in our study. The role of this and other proteins bound by SGO1-AS1 in gastric carcinoma deserves further investigation.

Identifying TGF $\beta$-induced ZEB1 as a potent transcriptional repressor of SGO1-AS1 is another important finding of this study. Here, we demonstrate that a reciprocal negative feedback loop exists between SGO1-AS1 and TGF $\beta / Z E B 1$. Although the double positive feedback loop between TGF $\beta$ and IncRNA is well documented [40-42], to the best of our knowledge, the reciprocal repressive loop between TGF $\beta$ and lncRNA has rarely been observed. Our current study provides evidence of a reciprocal repressive loop between TGF $\beta$ and the lncRNA SGO1-AS1 in GC metastasis. We show that ZEB1 induced by TGF $\beta$ transcriptionally inhibits SGO1-AS1 expression; in turn, SGO1-AS1 inhibits TGF $\beta$ expression by reducing TGFB mRNA stability, which mediates the reciprocal repressive loop between TGF $\beta / Z E B 1$ and SGO1-AS1 in GC.

TGF $\beta$ signaling is highly conserved in multicellular organisms and is involved in multiple cellular processes, such as cell growth, stemness, migration, invasion, the EMT, ECM, remodeling and immune regulation [43]. The activation of canonical TGF $\beta$ signaling is caused by the binding of TGF $\beta$ ligands (TGF $\beta 1$, TGF $\beta 2$ and TGF $\beta 3$ ) to heteromeric TGF $\beta$ type I and II receptors, which phosphorylate SMAD2 and SMAD3, resulting in complex formation with SMAD4 and nuclear translocation to regulate target gene transcription [44]. TGF $\beta$ plays a critical role in tumorigenesis and tumor progression in a complex and pleiotropic manner; in early tumor initiation, it plays a tumor-suppressive role by inhibiting cell proliferation and stimulating apoptosis; however, in advanced tumors, it promotes tumor progression by inducing the EMT, which is correlated with increased invasiveness, metastasis and chemoresistance in tumor cells $[45,46]$. Because of its role in advanced tumors, TGF $\beta$ is considered a therapeutic target. Several strategies have been proposed to inhibit TGF $\beta$ signaling to combat malignant tumors (e.g., small-molecule inhibitors of receptor kinases, TGF $\beta$ neutralizing antibodies and antisense

\footnotetext{
(See figure on next page.)

Fig. 7 TGF $\beta$ downregulates SGO1-AS1 transcription via ZEB1. a. qRT-PCR analyses of the SGO1-AS1, SNAI and ZEB1 levels in SGC7901 cells incubated with TGF $\beta 1$ ( $3 \mathrm{ng} / \mathrm{mL}$ or $10 \mathrm{ng} / \mathrm{mL})$ for $24 \mathrm{~h}$. b. Expression levels of SGO1-AS1, SNAl and ZEB1 in BGC823 and AGS cells stimulated with $10 \mathrm{ng} / \mathrm{mL}$ TGF $\beta 1$ for $24 \mathrm{~h} .{ }^{*} P<0.05$, ${ }^{* *} P<0.01$ and ${ }^{* * *} P<0.001$ compared to those without TGF $\beta 1$ stimulation. c. SGO1-AS1, SNAI and ZEB1 levels in SGC7901 cells incubated with SB431542 $(10 \mu \mathrm{M})$ for $24 \mathrm{~h} .{ }^{*} P<0.05,{ }^{*} P<0.01$. d. A schematic diagram illustrating the four putative ZEB1 binding sites (Sites A, B, C and D) in the SGO1-AS1 promoter. e. Luciferase reporter assays of the SGO1-AS1 promoter region containing either wild-type (WT) or mutated (Mut A, Mut B, Mut C, Mut D, or Mut A-D) ZEB1 binding sites without or with TGF $\beta 1$ exposure. ${ }^{*} P<0.05$, ${ }^{* *} P<0.01,{ }^{* * *} P<0.001$ and ns, not significant compared to without the TGF $\beta 1$ stimulation. f. SGO1-AS1 expression was examined by a qRT-PCR analysis in TGF $\beta 1$-treated SGC7901 and BGC823 cells transfected with ZEB1 siRNAs. Western blot analysis was performed to assess the inhibition efficiency in the same cells (right). ${ }^{*} P<0.01$. g. Upper: Putative ZEB1-binding sites on the SGO1-AS1 promoter region and design-indicated primers. Lower: ChIP analysis of ZEB1 enrichment on the SGO1-AS1 promoter in SGC7901 cells treated with TGF $\beta 1$. IgG and anti-GAPDH antibodies were used as controls. ${ }^{*} P<0.05$, ${ }^{* * *} P<0.001$. In all cases, error bars indicateSDs from three independent experiments
} 


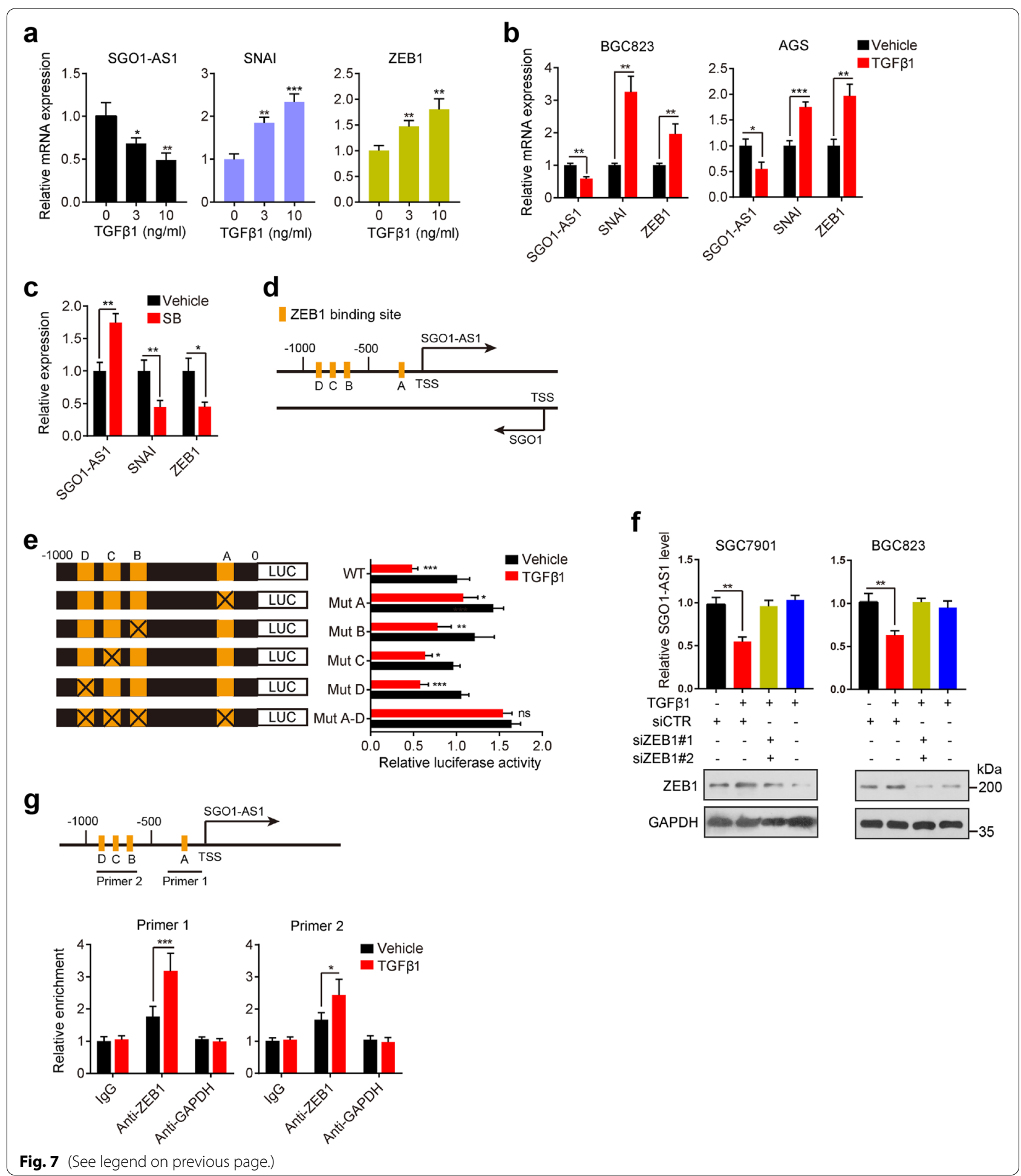

compounds) [47]. Our finding that SGO1-AS1 and TGF $\beta / Z E B 1$ form a double-negative feedback loop hints at the possibility of new therapeutic approaches to block the TGF $\beta$ signal by introducing SGO1-AS1 or using the 
a

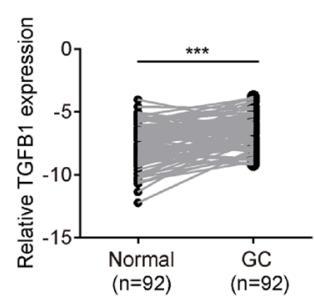

b

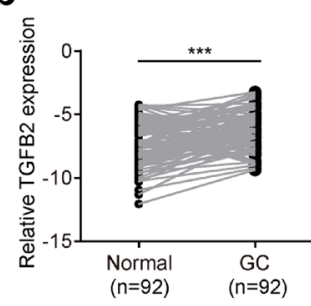

C
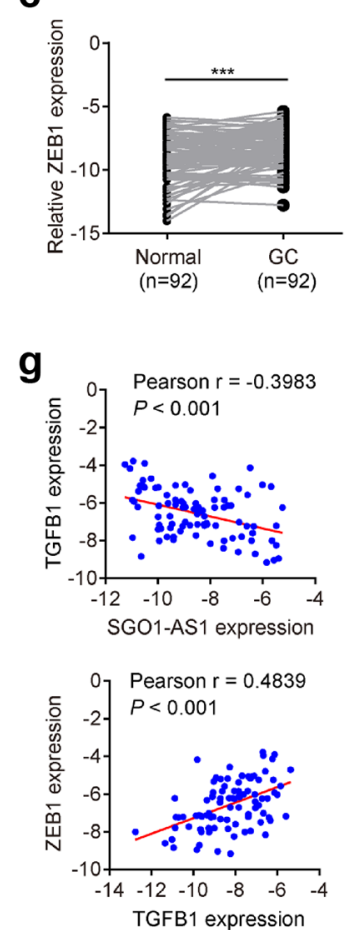

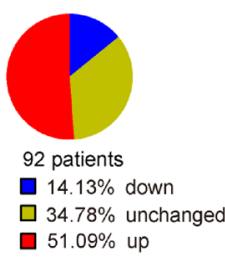

$\square 51.09 \%$ up

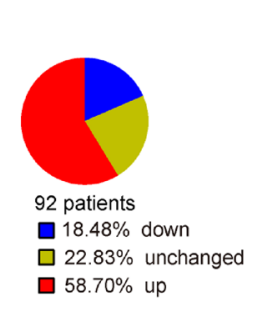

$\square 58.70 \%$ up

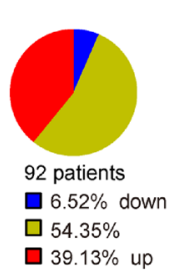

d
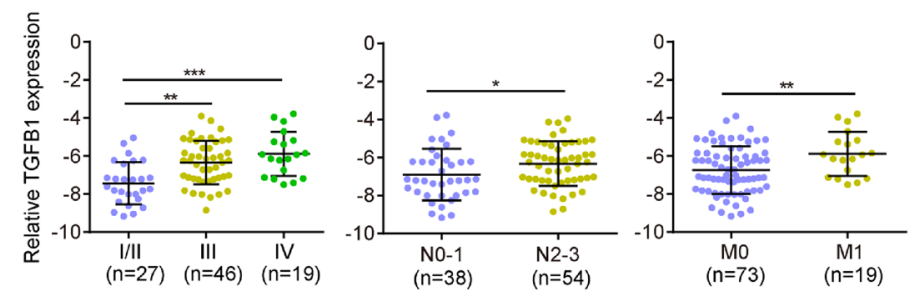

e
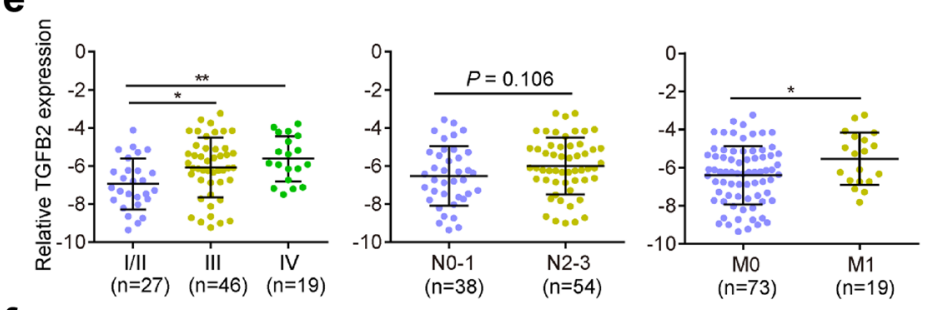

f
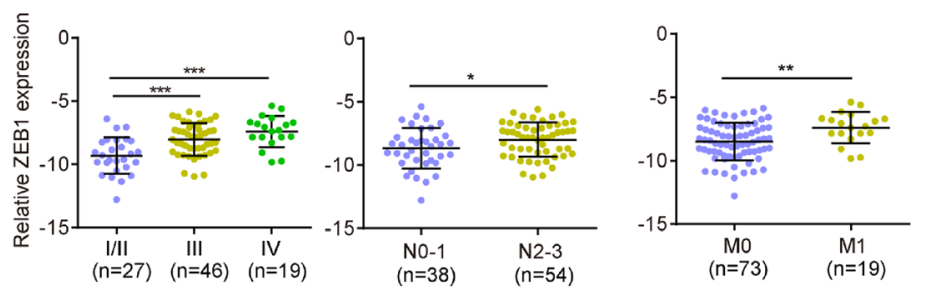

h

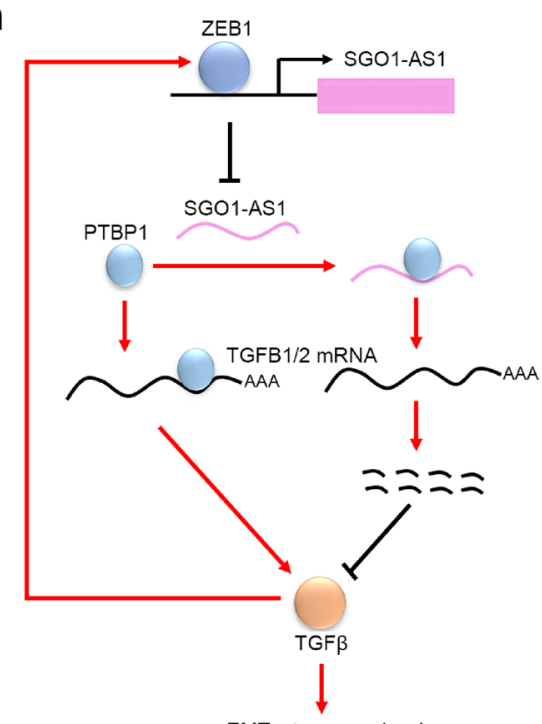

EMT, stemness, invsion and metastasis

Fig. 8 Expression levels of TGFB1/2 and ZEB1 and their correlations with SGO1-AS1 expression in GC tissues. a-c. Relative expression levels of TGFB1, TGFB2 and ZEB1 in 92 paired GC and normal tissues from Cohort 2 were quantified by qRT-PCR. The pie charts show the proportions of samples in the downregulation (blue), upregulation (red) and no change (yellow) categories. d-f. The expression levels of TGFB1, TGFB2 and ZEB1 in GCs according to their clinical stage and status of lymph node or distant metastasis. g. SGO1-AS1 expression was inversely correlated with TGFB1, TGFB2 and ZEB1 expression, while ZEB1 expression was positively correlated with TGFB1/2 expression in the GC specimens. Error bars indicate SDs. ${ }^{*} P<0.05,{ }^{* *} P<0.01,{ }^{* *} P<0.001$. h. Schematic illustration of the TGF $\beta / Z E B 1 / S G O 1-A S 1$ signaling pathway. TGF $\beta$ downregulates SGO1-AS1 by inducing ZEB1. SGO1-AS1 inactivates TGF $\beta$ signaling by promoting TGFB mRNA decay 
interference of ZEB1, although this possibility remains to be confirmed by future studies.

\section{Conclusions}

Our study identified a metastasis-suppressive lncRNA that functions as an endogenous inhibitor of the TGF $\beta$ pathway and suppresses GC metastasis and progression. Our data further highlight the importance of the doublenegative feedback loop between SGO1-AS1 and TGF $\beta$ / ZEB1 in GC metastasis. These findings provide novel information for understanding the mechanisms underlying the pathogenesis in GC metastasis and new insight into the potential use of SGO1-AS1-TGF $\beta$-ZEB1 for the development of new treatment strategies for GC.

\begin{abstract}
Abbreviations
IncRNAs: Long noncoding RNAs; GC: Gastric carcinoma; qRT-PCR: Quantitative reverse transcription polymerase chain reaction; ISH: In situ hybridization; EMT: Epithelial-to-mesenchymal transition; GEO: Gene expression omnibus; qPCR: Quantitative polymerase chain reaction; RACE: Rapid amplification of cDNA ends; DMEM: Dulbecco's modified eagle's medium; shRNA: Short hairpin RNA; sgRNA: Small guide RNA; SDS-PAGE: Sodium dodecyl sulfatepolyacrylamide gel electrophoresis; RIP: RNA immunoprecipitation; RNA-seq: RNA sequencing; ChIP: Chromatin immunoprecipitation; NC: Normal control; SD: Standard deviation; CPAT: Coding-potential assessment tool; CPC: Coding potential calculator; hnRNP: Heterogeneous nuclear ribonucleoprotein; RBP: RNA-binding protein; KO: Knockout; KEGG: Kyoto Encyclopedia of Genes and Genomes; ECM: Extracellular matrix; GSEA: Gene set enrichment analysis; TCGA :The cancer genome atlas; ACD: Actinomycin D; T $\beta R$ I: TGF $\beta$ type I receptor; EMT-TFs: EMT-inducing transcription factors; ELISA: Enzyme linked immunosorbent assay; WT: Wild-type.
\end{abstract}

\section{Supplementary Information}

The online version contains supplementary material available at https://doi. org/10.1186/s13046-021-02140-0.

Additional file 1: Fig. S1 SGO1-AS1 was downregulated in GC tissues. Expression levels of SGO1-AS1 and 12 other IncRNAs validated by qRT-PCR in 18 paired gastric cancer tissues and adjacent normal tissues (Cohort 1). The results are expressed as $-\Delta \mathrm{Ct}$. ${ }^{* *} P<0.05,{ }^{* *} P<0.01,{ }^{* * *} P<0.001$. Fig. S2 Characterization of human SGO1-AS1 as a long noncoding RNA. a. Schematic diagram of the genomic locus and isoforms of SGO1-AS1 in the UCSC Genome Browser (http://genome.ucsc.edu/). b. Identification of full-length SGO1-AS1 by $5^{\prime}$ and $3^{\prime}$ RACE. Left: Representative images of the PCR products from 5' RACE and 3' RACE. Right: Nucleotide sequence of full-length human SGO1-AS1. c. Coding potential of SGO1-AS1 as predicted by the CPAT and CPC tools. The InCRNA MALAT1 and the proteincoding genes GAPDH and ACTB are also shown. d. Relative distribution of SGO1-AS1 in gastric cell lines as determined by RT-PCR. SGO1-AS1 was mainly expressed in the cytoplasm of epithelial cells. Fig. S3 SGO1-AS1 inhibits GC cell migration and growth. a. qRT-PCR showed the relative levels of SGO1-AS1 expression in human gastric cancer cell lines compared to those in the immortalized human gastric epithelial cell line GES1. b. qRT-PCR analysis was used to confirm the level of SGO1-AS1 in cells with stable overexpression of SGO1-AS1. C. QRT-PCR analysis was performed to assess the inhibition efficiency in MKN28 cells infected with SGO1-AS1 shRNA lentiviruses targeting different regions of SGO1-AS1. d. Cell migration in BGC823 cells stably expressing SGO1-AS1 and control cells was examined by a wound healing assay. Scale bar, $150 \mu \mathrm{m}$. e. Cell proliferation assay in SGC7901 and BGC823 cells stably expressing SGO1-AS1, MKN28 cells stably silencing SGO1-AS1 and the respective control cells. f-g. A soft agar colony formation assay was carried out in the indicated cells. Scale bars, $100 \mu \mathrm{m}$. h. Overexpression of SGO1-AS1 inhibits GC tumor growth in a nude mouse model. SGC-7901 cells with stable overexpression of SGO1-AS1 or the control were inoculated subcutaneously into nude mice ( $n=6$ mice/group). The mice were sacrificed at 28 days postinoculation, and the tumors were weighed. Error bars indicate SD. ${ }^{*} P<0.05,{ }^{* *} P<0.01$, ${ }^{* * *} P<0.001$. Fig. S4 Effects of SGO1-AS1 on the expression of the sense gene SGO1. a-b. qRT-PCR and Western blotting analyses of the SGO1 levels in SGC7901 and BGC823 cells stably expressing SGO1-AS1, MKN-28 cells stably silencing SGO1-AS1 and the related control cells. Error bars indicate SDs from three independent experiments. ${ }^{*} P<0.05,{ }^{*} P<0.01$. Fig. S5 SGO1-AS1 reduces TGFB1/2 mRNA stability. a. Western blot analysis was used to evaluate PTBP1 expression in human gastric cancer cell lines and normalgastric epithelial cell line. b. qRT-PCR analysis was performed to examine the expression levels of genes related to the TGF $\beta$ pathway in SGC7901 cells transfected with PTBP1 shRNA or the control. C. PTBP1 RIP assay was performed to analyze the interactions between PTBP1 and TGFB1/2 or ID3 in SGC7901 cells. The relative fold enrichment of these mRNAs compared to lgG was determined by qRT-PCR. SMAD5 and GAPDH were used as negative controls. d. RIP-qPCR assay was used to evaluate the interaction between PTBP1 and ID2 mRNA in MKN28 cells with SGO1-AS1 knockdown or SGC7901 cells with SGO1-AS1 overexpression. e. Deletion mapping was performed to determine the binding domain of PTBP1 to TGFB1/2 mRNA using full-length or truncated PTBP1. f-g. mRNA (f) and protein ( $g$ ) expression levels of TGF $\beta 1 / 2$ in xenograft tumor tissues recovered from nude mice. \#1-3 denote individual tumors grown in different mice. h. Western blot analysis of AGO2, TGF $\beta 1$ and TGF $\beta 2$ in control and PTBP1-KO MKN28 cells transfected with siAGO2 or control siRNA. i. RIP-qPCR assay of the interaction between the AGO2 protein and TGFB1/2 mRNA in MKN28 cells with PTBP1 knockout or SGO1AS1 knockdown. Error bars are SDs from three independent experiments. ${ }^{*} P<0.05,{ }^{* *} P<0.01,{ }^{* * *} P<0.001$ and ns, not significant. Fig. S6 SGO1-AS1 changes cell morphology and suppresses cell invasion and stemness. a-c. Representative phase-contract (a), cell invasion (b) and tumor spheroid images (c) of the indicated cells are shown. a, Scale bars, $100 \mu \mathrm{m}$; b-c, Scale bars, $150 \mu \mathrm{m}$. Fig. S7 Expression level of PTBP1 and its correlation with SGO1-AS1, TGFB1/2 and ZEB1 expression in GC tissues. a. Relative expression levels of PTBP1 in 92 paired GC and normal tissues from Cohort 2 were quantified by qRT-PCR. The pie chart shows the proportions of samples in the downregulation (blue), upregulation (red) and no change (yellow) categories. b. The expression level of PTBP1 in GCs according to their clinical stage and status of lymph node or distant metastasis. C. Correlation between PTBP1 expression and SGO-AS1, TGFB1, TGFB2 and ZEB1 expression in GC specimens. a-C, Error bars indicate SDs. ${ }^{*} P<0.05$, ${ }^{* *} P<0.01,{ }^{* * *} P<0.001$ and ns, not significant. Table S1. Clinicopathological characteristics of Cohort 2 samples. Table S2. Clinicopathological characteristics of Cohort 3 samples. Table S3. Primers used for quantitative PCR. Table S4. Primers for rapid amplification of CDNA ends analysis. Table S5. Correlation between clinicopathological parameters and SGO1AS1 levels in 95 cases of GC tissues (Cohort 3). Table S6. Univariate and multivariate analyses of factors associated with overall survival. Table S7. Mass spectrometry protein identification results for biotinylated SGO1-AS1 RNA pull down.

\section{Acknowledgments}

Not applicable.

\section{Authors' contributions}

$\mathrm{DH}, \mathrm{KZ}$ and $\mathrm{MD}$ designed the study. MD, YG and JX supervised the study. DH, $K Z, Y G, W Z, R Z$ and $Y X$ performed the experiments. JC, DH and YL conducted bioinformatics analysis and experiment data analyses. ND collected tissue specimens and clinical data. MD and JX wrote the manuscript. All authors read and approved the final manuscript.

\section{Funding}

This work was supported by the National Natural Science Foundation of China (No. 81972771, No. 81672452 and No. 81472625), National Natural Science Foundation of Guangdong Province (No. 2018B0303110015 and No. 2018A0303130314) and Guangdong Medical Research Foundation (No. B2018283). 


\section{Availability of data and materials}

The microarray data have been deposited in the GEO data base under accession numbers GSE157289, GSE157582 and GSE157941.

\section{Declarations}

\section{Ethics approval and consent to participate}

All the procedures carried out in the research involving human participants are in accordance with the ethical standards of the Institutional Review Board of Affiliated Cancer Hospital of Guangzhou Medical University. Each participant signed an informed consent before participating to this study. All animal studies were approved by the Institutional Animal Care and Use Committee of Guangzhou Medical University, and all animals were ethically and humanely treated.

\section{Consent for publication}

All authors agreed on the manuscript.

\section{Competing interests}

The authors declare no conflict of interest.

\section{Author details}

${ }^{1}$ Affiliated Cancer Hospital \& Institute of Guangzhou Medical University, No.78 Hengzhigang Road, Guangzhou 510095, Guangdong, China. ${ }^{2}$ Department of Clinical Laboratory, Sun Yat-Sen University Cancer Center, State Key Laboratory of Oncology in South China, Guangzhou, China. ${ }^{3}$ Department of Oncology, The Fifth Affiliated Hospital, Guangzhou Medical University, Guangzhou, China. ${ }^{4}$ Department of Laboratory, Guangzhou Women and Children's Medical Centre, Guangzhou Medical University, Guangzhou, China. ${ }^{5}$ Laboratory of Oncology Science and Molecular Biology, ShunDe Hospital of Guangzhou University of Chinese Medicine, No.12 Jinsha Avenue, Shunde District, Foshan 528333, Guangdong, China.

Received: 13 May 2021 Accepted: 12 October 2021

Published online: 28 October 2021

\section{References}

1. Sung H, Ferlay J, Siegel RL, Laversanne M, Soerjomataram I, Jemal A, et al. Global cancer statistics 2020: GLOBOCAN estimates of incidence and mortality worldwide for 36 cancers in 185 countries. CA Cancer J Clin. 2021;71(3):209-49.

2. Bernards N, Creemers GJ, Nieuwenhuijzen GA, Bosscha K, Pruijt JF, Lemmens VE. No improvement in median survival for patients with metastatic gastric cancer despite increased use of chemotherapy. Ann Oncol. 2013;24(12):3056-60.

3. Ponting $\mathrm{CP}$, Oliver PL, Reik W. Evolution and functions of long noncoding RNAs. Cell. 2009;136(4):629-41.

4. Derrien T, Guigo R, Johnson R. The long non-coding RNAs: a new (P)layer in the "dark matter". Front Genet. 2011;2:107.

5. Kopp F, Mendell JT. Functional classification and experimental dissection of long noncoding RNAs. Cell. 2018;172(3):393-407.

6. Schonrock N, Harvey RP, Mattick JS. Long noncoding RNAs in cardiac development and pathophysiology. Circ Res. 2012;111(10):1349-62.

7. Liu HT, Liu S, Liu L, Ma RR, Gao P. EGR1-mediated transcription of IncRNA-HNF1A-AS1 promotes cell-cycle progression in gastric Cancer. Cancer Res. 2018;78(20):5877-90.

8. Xu MD, Wang Y, Weng W, Wei P, Qi P, Zhang Q, et al. A positive feedback loop of IncRNA-PVT1 and FOXM1 facilitates gastric Cancer growth and invasion. Clin Cancer Res. 2017;23(8):2071-80.

9. Sun TT, He J, Liang Q, Ren LL, Yan TT, Yu TC, et al. LncRNA GCInc1 promotes gastric carcinogenesis and may act as a modular scaffold of WDR5 and KAT2A complexes to specify the histone modification pattern. Cancer Discov. 2016;6(7):784-801.

10. Zhuo W, Liu Y, Li S, Guo D, Sun Q, Jin J, et al. Long noncoding RNA GMAN, up-regulated in gastric Cancer tissues, is associated with metastasis in patients and promotes translation of Ephrin A1 by competitively binding GMAN-AS. Gastroenterology. 2019;156(3):676-91 e11.
11. He W, Liang B, Wang C, Li S, Zhao Y, Huang Q, et al. MSC-regulated IncRNA MACC1-AS1 promotes stemness and chemoresistance through fatty acid oxidation in gastric cancer. Oncogene. 2019;38(23):4637-54.

12. Hu Y, Wang J, Qian J, Kong X, Tang J, Wang Y, et al. Long noncoding RNA GAPLINC regulates CD44-dependent cell invasiveness and associates with poor prognosis of gastric cancer. Cancer Res. 2014;74(23):6890-902.

13. Wang L, Park HJ, Dasari S, Wang S, Kocher JP, Li W. CPAT: coding-potential assessment tool using an alignment-free logistic regression model. Nucleic Acids Res. 2013:41(6):e74.

14. Kong L, Zhang Y, Ye ZQ, Liu XQ, Zhao SQ, Wei L, et al. CPC: assess the protein-coding potential of transcripts using sequence features and support vector machine. Nucleic Acids Res. 2007;35(Web Server issue):W345-9.

15. Vuong JK, Lin CH, Zhang M, Chen L, Black DL, Zheng S. PTBP1 and PTBP2 serve both specific and redundant functions in neuronal pre-mRNA splicing. Cell Rep. 2016;17(10):2766-75.

16. Calabretta S, Bielli P, Passacantilli I, Pilozzi E, Fendrich V, Capurso G, et al. Modulation of PKM alternative splicing by PTBP1 promotes gemcitabine resistance in pancreatic cancer cells. Oncogene. 2016:35(16):2031-9.

17. Zhang L, Yang Z, Trottier J, Barbier O, Wang L. Long noncoding RNA MEG3 induces cholestatic liver injury by interaction with PTBP1 to facilitate shp mRNA decay. Hepatology. 2017;65(2):604-15.

18. Monzon-Casanova E, Screen M, Diaz-Munoz MD, Coulson RMR, Bell SE, Lamers $\mathrm{G}$, et al. The RNA-binding protein PTBP1 is necessary for B cell selection in germinal centers. Nat Immunol. 2018;19(3):267-78.

19. Matus-Nicodemos R, Vavassori S, Castro-Faix M, Valentin-Acevedo A Singh $\mathrm{K}$, Marcelli $\mathrm{V}$, et al. Polypyrimidine tract-binding protein is critical for the turnover and subcellular distribution of CD40 ligand mRNA in CD4+ T cells. J Immunol. 2011;186(4):2164-71.

20. Sawicka K, Bushell M, Spriggs KA, Willis AE. Polypyrimidine-tract-binding protein: a multifunctional RNA-binding protein. Biochem Soc Trans. 2008;36(Pt 4):641-7.

21. Sun YM, Wang WT, Zeng ZC, Chen TQ, Han C, Pan Q, et al. circMYBL2, a circRNA from MYBL2, regulates FLT3 translation by recruiting PTBP1 to promote FLT3-ITD AML progression. Blood. 2019;134(18):1533-46.

22. Bielli P, Panzeri V, Lattanzio R, Mutascio S, Pieraccioli M, Volpe E, et al. The splicing factor PTBP1 promotes expression of oncogenic splice variants and predicts poor prognosis in patients with non-muscle-invasive bladder Cancer. Clin Cancer Res. 2018:24(21):5422-32.

23. Cui J, Placzek WJ. PTBP1 modulation of MCL1 expression regulates cellula apoptosis induced by antitubulin chemotherapeutics. Cell Death Differ. 2016;23(10):1681-90

24. Pina JM, Reynaga JM, Truong AAM, Keppetipola NM. Post-translationa modifications in Polypyrimidine tract binding proteins PTBP1 and PTBP2. Biochemistry. 2018;57(26):3873-82.

25. Kawamata T, Tomari Y. Making RISC. Trends Biochem Sci. 2010;35(7):368-76.

26. Makarova JA, Shkurnikov MU, Wicklein D, Lange T, Samatov TR, Turchinovich AA, et al. Intracellular and extracellular microRNA: an update on localization and biological role. Prog Histochem Cytochem. 2016;51(3-4):33-49.

27. Chiang SP, Cabrera RM, Segall JE. Tumor cell intravasation. Am J Physiol Cell Physiol. 2016;311(1):C1-C14.

28. Welm AL. TGFbeta primes breast tumor cells for metastasis. Cell. 2008;133(1):27-8

29. Yuan JH, Yang F, Wang F, Ma JZ, Guo YJ, Tao QF, et al. A long noncoding RNA activated by TGF-beta promotes the invasion-metastasis cascade in hepatocellular carcinoma. Cancer Cell. 2014;25(5):666-81.

30. Larsen JE, Nathan V, Osborne JK, Farrow RK, Deb D, Sullivan JP, et al. ZEB1 drives epithelial-to-mesenchymal transition in lung cancer. J Clin Invest. 2016:126(9):3219-35.

31. Wu HT, Zhong HT, Li GW, Shen JX, Ye QQ, Zhang ML, et al. Oncogenic functions of the EMT-related transcription factor ZEB1 in breast cancer. $J$ Transl Med. 2020;18(1):51.

32. Bhan A, Soleimani M, Mandal SS. Long noncoding RNA and Cancer: a new paradigm. Cancer Res. 2017;77(15):3965-81.

33. Xie R, Chen X, Chen Z, Huang M, Dong W, Gu P, et al. Polypyrimidine tract binding protein 1 promotes lymphatic metastasis and proliferation of bladder cancer via alternative splicing of MEIS2 and PKM. Cancer Lett. 2019:449:31-44.

34. Georgilis A, Klotz S, Hanley CJ, Herranz N, Weirich B, Morancho B, et al. PTBP1-mediated alternative splicing regulates the inflammatory 
Secretome and the pro-tumorigenic effects of senescent cells. Cancer Cell. 2018;34(1):85-102 e9.

35. Liu C, Yang Z, Wu J, Zhang L, Lee S, Shin DJ, et al. Long noncoding RNA H19 interacts with polypyrimidine tract-binding protein 1 to reprogram hepatic lipid homeostasis. Hepatology. 2018;67(5):1768-83.

36. Jiang J, Chen X, Liu H, Shao J, Xie R, Gu P, et al. Polypyrimidine tract-binding protein 1 promotes proliferation, migration and invasion in clear-cell renal cell carcinoma by regulating alternative splicing of PKM. Am J Cancer Res. 2017:7(2):245-59.

37. Ramos AD, Andersen RE, Liu SJ, Nowakowski TJ, Hong SJ, Gertz C, et al. The long noncoding RNA Pnky regulates neuronal differentiation of embryonic and postnatal neural stem cells. Cell Stem Cell. 2015;16(4):439-47.

38. Liu J, Li Y, Tong J, Gao J, Guo Q, Zhang L, et al. Long non-coding RNAdependent mechanism to regulate heme biosynthesis and erythrocyte development. Nat Commun. 2018;9(1):4386.

39. Huan L, Guo T, Wu Y, Xu L, Huang S, Xu Y, et al. Hypoxia induced LUCAT1/ PTBP1 axis modulates cancer cell viability and chemotherapy response. Mol Cancer. 2020;19(1):11.

40. Sakai S, Ohhata T, Kitagawa K, Uchida C, Aoshima T, Niida H, et al. Long noncoding RNA ELIT-1 acts as a Smad3 cofactor to facilitate TGFbeta/ Smad signaling and promote epithelial-mesenchymal transition. Cancer Res. 2019;79(11):2821-38.
41. Zhang K, Han X, Zhang Z, Zheng L, Hu Z, Yao Q, et al. The liver-enriched Inc-LFAR1 promotes liver fibrosis by activating TGFbeta and notch pathways. Nat Commun. 2017;8(1):144

42. Lang C, Dai Y, Wu Z, Yang Q, He S, Zhang X, et al. SMAD3/SP1 complexmediated constitutive active loop between IncRNA PCAT7 and TGF-beta signaling promotes prostate cancer bone metastasis. Mol Oncol. 2020;14(4):808-28.

43. Derynck R, Zhang YE. Smad-dependent and Smad-independent pathways in TGF-beta family signalling. Nature. 2003:425(6958):577-84.

44. Shi Y, Massague J. Mechanisms of TGF-beta signaling from cell membrane to the nucleus. Cell. 2003;113(6):685-700.

45. Roberts $A B$, Wakefield $L M$. The two faces of transforming growth factor beta in carcinogenesis. Proc Natl Acad Sci U S A. 2003;100(15):8621-3.

46. Massague J. TGFbeta in Cancer. Cell. 2008;134(2):215-30.

47. Akhurst RJ, Hata A. Targeting the TGFbeta signalling pathway in disease. Nat Rev Drug Discov. 2012;11(10):790-811.

\section{Publisher's Note}

Springer Nature remains neutral with regard to jurisdictional claims in published maps and institutional affiliations.
Ready to submit your research? Choose BMC and benefit from:

- fast, convenient online submission

- thorough peer review by experienced researchers in your field

- rapid publication on acceptance

- support for research data, including large and complex data types

- gold Open Access which fosters wider collaboration and increased citations

- maximum visibility for your research: over $100 \mathrm{M}$ website views per year

At BMC, research is always in progress.

Learn more biomedcentral.com/submissions 